\title{
Effective Interactive Resolution of Static Analysis Alarms
}

\author{
XIN ZHANG, Georgia Institute of Technology, USA \\ RADU GRIGORE, University of Kent, UK \\ XUJIE SI, University of Pennsylvania, USA \\ MAYUR NAIK, University of Pennsylvania, USA
}

\begin{abstract}
We propose an interactive approach to resolve static analysis alarms. Our approach synergistically combines a sound but imprecise analysis with precise but unsound heuristics, through user interaction. In each iteration, it solves an optimization problem to find a set of questions for the user such that the expected payoff is maximized. We have implemented our approach in a tool, URSA, that enables interactive alarm resolution for any analysis specified in the declarative logic programming language Datalog. We demonstrate the effectiveness of UrSA on a state-of-the-art static datarace analysis using a suite of 8 Java programs comprising 41-194 KLOC each. URSA is able to eliminate $74 \%$ of the false alarms per benchmark with an average payoff of $12 \times$ per question. Moreover, URSA prioritizes user effort effectively by posing questions that yield high payoffs earlier.
\end{abstract}

CCS Concepts: • Theory of computation $\rightarrow$ Program analysis; Program verification;

Additional Key Words and Phrases: Program Analysis, Alarm Resolution, Generalization, Prioritization

\section{ACM Reference Format:}

Xin Zhang, Radu Grigore, Xujie Si, and Mayur Naik. 2017. Effective Interactive Resolution of Static Analysis Alarms. Proc. ACM Program. Lang. 1, OOPSLA, Article 57 (October 2017), 30 pages.

https://doi.org/10.1145/3133881

\section{INTRODUCTION}

Automated static analyses make a number of approximations. These approximations are necessary because the static analysis problem is undecidable in general [Rice 1953]. However, they result in many false alarms in practice, which in turn imposes a steep burden on users of the analyses.

A common pragmatic approach to reduce false alarms involves applying heuristics to suppress their root causes. For instance, such a heuristic may ignore a certain code construct that can lead to a high false alarm rate [Bessey et al. 2010]. While effective in alleviating user burden, however, such heuristics potentially render the analysis results unsound.

In this paper, we propose a novel methodology that synergistically combines a sound but imprecise analysis with precise but unsound heuristics, through user interaction. Our key idea is that, instead of directly applying a given heuristic in a manner that may unsoundly suppress false alarms, the combined approach poses questions to the user about the truth of root causes that are targeted by the heuristic. If the user confirms them, only then is the heuristic applied to eliminate the false alarms, with the user's knowledge.

To be effective, however, the combined approach must accomplish two key goals: generalization and prioritization. We describe each of these goals and how we realize them.

Permission to make digital or hard copies of all or part of this work for personal or classroom use is granted without fee provided that copies are not made or distributed for profit or commercial advantage and that copies bear this notice and the full citation on the first page. Copyrights for components of this work owned by others than the author(s) must be honored Abstracting with credit is permitted. To copy otherwise, or republish, to post on servers or to redistribute to lists, requires prior specific permission and/or a fee. Request permissions from permissions@acm.org.

(C) 2017 Copyright held by the owner/author(s). Publication rights licensed to Association for Computing Machinery.

2475-1421/2017/10-ART57

https://doi.org/10.1145/3133881

Proc. ACM Program. Lang., Vol. 1, No. OOPSLA, Article 57. Publication date: October 2017. 
Generalization. The number of questions posed to the user by our approach should be much smaller compared to the number of false alarms that are eliminated. Otherwise, the effort in answering those questions could outweigh the effort in resolving the alarms directly. To realize this objective for analyses that produce many alarms, we observe that most alarms are often symptoms of a relatively small set of common root causes. For example, a method that is falsely deemed reachable can result in many false alarms in the body of that method. Inspecting this single root cause is easier than inspecting multiple alarms.

Prioritization. Since the number of false alarms resulting from different root causes may vary, the user might wish to only answer questions with relatively high payoffs. Our approach realizes this objective by interacting with the user in an iterative manner instead of posing all questions at once. In each iteration, the questions asked are chosen such that the expected payoff is maximized. Note that the user may be asked multiple questions per iteration because single questions may be insufficient to resolve any of the remaining alarms. Asking questions in order of decreasing payoff allows the user to stop answering them when diminishing returns set in. Finally, the iterative process allows incorporating the user's answers to past questions in making better choices of future questions, and thereby further amplify the reduction in user effort.

We formulate the problem to be solved in each iteration of our approach as a non-linear optimization problem called the optimum root set problem. This problem aims at finding a set of questions that maximizes the benefit-cost ratio in terms of the number of false alarms expected to be eliminated and the number of questions posed. We propose an efficient solution to this problem by reducing it to a sequence of integer linear programming (ILP) instances. Each ILP instance encodes the dependencies between analysis facts, and weighs the benefits and costs of questioning different sets of root causes. Since our objective function is non-linear, we solve a sequence of ILP instances that performs a binary search between the upper bound and lower bound of the benefit-cost ratio.

Our approach automatically generates the ILP formulation for any analysis specified in a constraint language. Specifically, we target Datalog, a declarative logic programming language that is widely used in formulating program analyses [Madsen et al. 2016; Mangal et al. 2015; Smaragdakis and Bravenboer 2010; Smaragdakis et al. 2014; Whaley et al. 2005; Zhang et al. 2014]. Our constraint-based approach also allows incorporating orthogonal techniques to reduce user effort, such as alarm clustering techniques that express dependencies between different alarms using constraints, possibly in a different abstract domain [Le and Soffa 2010; Lee et al. 2012].

We have implemented our approach in a tool called URSA and evaluate it on a static datarace analysis using a suite of 8 Java programs comprising 41-194 KLOC each. URSA eliminates $74 \%$ of the false alarms per benchmark with an average payoff of $12 \times$ per question. Moreover, URSA effectively prioritizes questions with high payoffs. Further, based on data collected from 40 Java programmers, we observe that the average time to resolve a root cause is only half of that to resolve an alarm. Together, these results show that our approach achieves significant reduction in user effort.

We summarize our contributions below:

(1) We propose a new paradigm to synergistically combine a sound but imprecise analysis with precise but unsound heuristics, through user interaction.

(2) We present an interactive algorithm that implements the paradigm. In each iteration, it solves the optimum root set problem which finds a set of questions with the highest expected payoff to pose to the user.

(3) We present an efficient solution to the optimum root set problem based on integer linear programming for a general class of constraint-based analyses.

(4) We empirically show that our approach eliminates a majority of the false alarms by asking only a few questions. Moreover, it effectively prioritizes questions with high payoffs. 


\section{Input Relations:}

$\operatorname{access}(p, o)$
$\operatorname{alias}\left(p_{1}, p_{2}\right)$
$\operatorname{escape}(o)$
$\operatorname{parallel}\left(p_{1}, p_{2}\right)$
unguarded $\left(p_{1}, p_{2}\right)$

Output Relations: $\operatorname{shared}(p)$ $\operatorname{race}\left(p_{1}, p_{2}\right)$ (program point $p$ accesses some field of abstract object $o$ ) (program points $p_{1}$ and $p_{2}$ access same memory location) (abstract object $o$ is thread-shared) (program points $p_{1}$ and $p_{2}$ can be reached by different threads simultaneously) (program points $p_{1}$ and $p_{2}$ are not guarded by any common lock)

(program point $p$ accesses thread-shared memory location) (datarace between program points $p_{1}$ and $p_{2}$ )

Rules:

$\operatorname{shared}(p):-\operatorname{access}(p, o)$, escape $(o)$.

$\operatorname{race}\left(p_{1}, p_{2}\right):-\operatorname{shared}\left(p_{1}\right), \operatorname{shared}\left(p_{2}\right), \operatorname{alias}\left(p_{1}, p_{2}\right), \operatorname{parallel}\left(p_{1}, p_{2}\right)$, unguarded $\left(p_{1}, p_{2}\right)$.

Fig. 2. Simplified static datarace analysis for Java in Datalog.

are marked in bold in Figure 1. Both fields are initialized in the constructor of RequestHandler which is invoked by the Server thread on line 25. Then, they are accessed in the run method by the Worker thread. Finally, they are accessed and set to null in the close method which is invoked to clean up the state of RequestHandler when the current connection is about to be closed. The close method can be either invoked in the service method by the Worker thread when the thread finishes processing the connection, or be invoked by the Java GUI thread in the stop method (on line 35) when the administrator clicks the stopButton to shut down the entire server by invoking stop (on line 16). The accesses in close are guarded by a synchronization block (on line 69-75) to prevent dataraces between them.

The program has multiple harmful race conditions: one on controlsocket between line 72 and line 57, and three on request between line 74 and line 55, 63, and 65. More concretely, these two fields can be accessed simultaneously by the Worker thread in run and the Java GUI thread in close. The race conditions can be fixed by guarding lines 55-66 with a synchronization block that holds a lock on the this object.

We next describe using a static analysis to detect these race conditions. In our implementation, we use the static datarace analysis from Chord [Naik 2006], which is context- and flow-sensitive, and combines a thread-escape analysis, a may-happen-in-parallel analysis, and a lockset analysis. Here, for ease of exposition, we use a much simpler version of that analysis, shown in Figure 2. It comprises two logical inference rules in Datalog. Rule (1) states that the instruction at program point $p$ accesses a thread-shared memory location if the instruction accesses a field of an object $o$, and $o$ is thread-shared. Since these properties are undecidable, the rule over-approximates them using abstract objects o, such as object allocation sites. Rule (2) uses the thread-escape information computed by Rule (1) along with several input relations to over-approximate dataraces: instructions at $p_{1}$ and $p_{2}$, at least one of which is a write, may race if a) each of them may access a thread-shared memory location, b) both of them may access the same memory location, c) they may be reached from different threads simultaneously, and d) they are not guarded by any common lock. All input relations are in fact calculated by the analysis itself but we treat them as inputs for brevity.

Since the analysis is over-approximating, it not only successfully captures the four real dataraces but also reports nine false alarms. The derivation of these false alarms is shown by the graph in Figure 3. We use line numbers of the program to identify program points $p$ and object allocation sites $o$. Each hyperedge in the graph is an instantiation of an analysis rule. In the graph, we elide tuples from all input relations except the ones in escape. For example, the dotted hyperedge is an instantiation of Rule (2): it derives race $(48,74)$, a race between lines 48 and 74 , from facts 


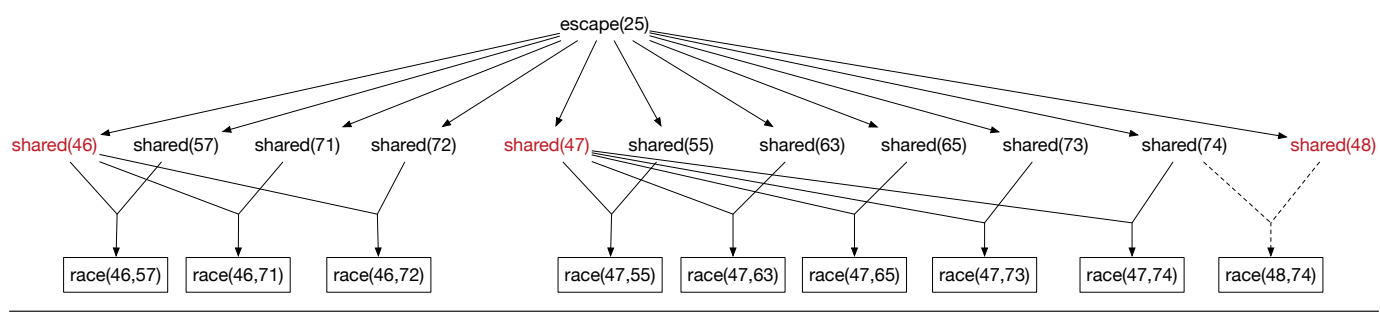

Fig. 3. Derivation of dataraces in example program. We only show parts related to false alarms for brevity.

shared(48), shared(74), alias $(48,74)$, parallel $(48,74)$, and unguarded $(48,74)$, which in turn are themselves derived or input facts.

Note that the above analysis does not report any datarace between accesses in the constructor of RequestHandler. This is because, by excluding tuples such as parallel $(46,46)$, the parallel relation captures the fact that the constructor can be only invoked by the Server thread. Likewise, it does not report any datarace between accesses in the close method as it is able to reason about locks via the unguarded relation. However, there are still nine false alarms among the 13 reported alarms. To find the four real races, the user must inspect all 13 alarms in the worst case.

To reduce the false alarm rate, Chord incorporates various heuristics, one of which is particularly effective in reducing false alarms in our scenario. This heuristic can be turned on by running Chord with option chord. datarace. exclude. init=true, which causes Chord to ignore all races that involve at least one instruction in an object constructor. Internally, the heuristic suppresses all shared tuples whose instruction lies in an object constructor. Intuitively, most of such memory accesses are on the object being constructed which typically stays thread-local until the constructor returns. Indeed, in our scenario, shared(46), shared(47), and shared(48) are all spurious (as the object only becomes thread-shared on line 27) and lead to nine false alarms. However, applying the heuristic can render the analysis result unsound. For instance, the object under construction can become thread-shared inside the constructor by being assigned to the field of a thread-shared object or by starting a new thread on it. Applying the heuristic can suppress true alarms that are derived from shared tuples related to such facts.

We present a new methodology and tool, URsa, to address this problem. Instead of directly applying the above heuristic in a manner that may introduce false negatives, URSA poses questions to the user about root causes that are targeted by the heuristic. In our example, such causes are the shared tuples in the constructor of RequestHandler. If the user confirms such a tuple as spurious, only then it is suppressed in the analysis, thereby eliminating all false alarms resulting from it.

URsa has two features that make it effective. First, it can eliminate a large number of false alarms by asking a few questions. The key insight is that most alarms are often symptoms of a relatively small set of common root causes. For instance, false alarms race $(47,55)$, race $(47,63)$, race $(47,65)$, race $(47,73)$, and race $(47,74)$ are all derived due to shared(47). Inspecting this single root cause is easier than inspecting all four alarms.

Second, URSA interacts with the user in an iterative manner, and prioritizes questions with high payoffs in earlier iterations. This allows the user to only answer questions with high payoffs and stop the interaction when the gain in alarms resolved has diminished compared to the effort in answering further questions.

URSA realizes the above two features by solving an optimization problem called the optimum root set problem, in each iteration. Recall that Chord's heuristic identified as potentially spurious the tuples shared(46), shared(47), shared(48); these appear in red in the derivation graph. We wish to ask the user if these tuples are indeed spurious, but not in an arbitrary order, because a few 
answers to well-chosen questions may be sufficient. We wish to find a small non-empty subset of those tuples, possibly just one tuple, which could rule out many false alarms. We call such a subset of tuples a root set, as the tuples in it are root causes for the potential false alarms, according to the heuristic. Each root set has an expected gain (how many false alarms it could rule out) and a cost (its size, which is how many questions the user will be asked). The optimum root set problem is to find a root set that maximizes the expected gain per unit cost. We refer to the gain per unit cost as the payoff. We next describe each iteration of URSA on our example. Here, we assume the user always answers the question correctly. Later we discuss the case where the user occasionally gives incorrect answers (Section 4.7 and Section 6.2).

Iteration 1. URSA picks $\{$ shared(47)\} as the optimum root set since it has an expected payoff of 5 , which is the highest among those of all root sets. Other root sets like \{shared(46), shared(47)\} may resolve more alarms but end up with lower expected payoffs due to larger numbers of required questions.

Based on the computed root set, UnsA poses a single question about shared(47) to the user: "Does line 47 access any thread-shared memory location? If the answer is no, five races will be suppressed as false alarms." Here URSA only asks one question, but there are other cases in which it may ask multiple questions in an iteration depending on the size of the computed optimum root set. Besides the root set, we also present the corresponding number of false alarms expected to be resolved. This allows the user to decide whether to answer the questions by weighing the gains in reducing alarms against the costs in answering them.

Suppose the user decides to continue and answers no. Doing so labels shared(47) as false, which in turn resolves five race alarms race $(47,55)$, $\operatorname{race}(47,63)$, $\operatorname{race}(47,65)$, race $(47,73)$, and race $(47,74)$, highlighting the ability of URSA to generalize user input. Next, URSA repeats the above process, but this time taking into account the fact that shared(47) is labeled as false.

Iteration 2. In this iteration, we have two candidates for the optimum root set: $\{\operatorname{shared}(46)\}$ with an expected payoff of 3 and $\{$ shared(48)\} with an expected payoff of 1 . While resolving $\{$ shared(46)\} is expected to eliminate $\{\operatorname{race}(46,57), \operatorname{race}(46,71)$, $\operatorname{race}(46,72)\}$, resolving $\{\operatorname{shared}(48)\}$ can only eliminate $\{$ race $(48,74)\}$.

URSA picks \{shared(46)\} as the optimum root set and poses the following question to the user: "Does line 46 access any thread-shared memory location? If the answer is no, three races will be suppressed as false alarms." Compared to the previous iteration, the expected payoff of the optimum root set has reduced, highlighting URSA's ability to prioritize questions with high payoffs.

We assume the user answers no, which labels shared(46) as false. As a result, race alarms race $(46,57)$, race $(46,71)$, and race $(46,72)$ are eliminated. At the end of this iteration, eight out of the 13 alarms have been eliminated. Moreover, only one false alarm race $(48,74)$ remains.

Iteration 3. In this iteration, there is only one root set $\{$ shared(48)\} which has an expected payoff of 1 . Similar to the previous two iterations, URSA poses a single question about shared(48) to the user along with the number of false alarms expected to be suppressed. At this point, the user may prefer to resolve the remaining five alarms manually, as the expected payoff is too low and very few alarms remain unresolved. URsA terminates if the user makes this decision. Otherwise, the user proceeds to answer the question regarding shared(48) and therefore resolve the only remaining false alarm race $(48,74)$. At this point, URSA terminates as all three tuples targeted by the heuristic have been answered.

In summary, URSA resolves, in a sound manner, 8 (or 9) out of 9 false alarms by only asking two (or three) questions. Moreover, it prioritizes questions with high payoffs in earlier iterations. 


$$
\begin{array}{lllll}
C::=\bar{c} & \text { (program) } & c::=l:-\bar{l} & \text { (constraint) } \\
l::=r(\bar{a}) & \text { (literal) } & a::=v \mid d & \text { (argument) }
\end{array}
$$

(a) Syntax of Datalog (overline means list).

$$
\begin{array}{llll}
r \in \mathbf{R}=\{a, \mathrm{~b}, \ldots\} & \text { (relation) } & \sigma \in \mathbf{V} \rightarrow \mathbf{D} & \text { (substitution) } \\
v \in \mathbf{V}=\{x, y, \ldots\} & \text { (variable) } & a \in \mathbf{A} \subseteq \mathbf{T} & \text { (alarms) } \\
d \in \mathbf{D}=\{0,1, \ldots\} & \text { (constant) } & q \in \mathbf{Q} \subseteq \mathbf{T} & \text { (potential causes) } \\
t \in \mathbf{T}=\mathbf{R} \times \mathbf{D}^{*} & \text { (tuple) } & f \in \mathbf{F} \subseteq \mathbf{Q} & \text { (causes) }
\end{array}
$$

(b) Auxiliary definitions and notations.

$$
\begin{aligned}
\llbracket C \rrbracket & :=\text { Ifp } S_{C} \\
S_{C}(T) & :=T \cup\left\{t \mid t \in s_{c}(T) \text { and } c \in C\right\} \\
s_{l_{0}:-l_{1}, \ldots, l_{n}}(T) & :=\left\{\sigma\left(l_{0}\right) \mid \sigma\left(l_{1}\right) \in T, \ldots, \sigma\left(l_{n}\right) \in T\right\}
\end{aligned}
$$

(c) Semantics of Datalog.

$$
\begin{aligned}
\llbracket C \rrbracket_{\mathrm{F}} & :=\operatorname{Ifp} S F_{C}^{\mathrm{F}} \\
S F_{C}^{\mathrm{F}}(T) & :=S_{C}(T) \backslash \mathbf{F}
\end{aligned}
$$

(d) Augmented semantics of Datalog.

Fig. 4. Syntax and semantics of Datalog without and with causes.

\section{THE OPTIMUM ROOT SET PROBLEM}

In this section, we define the Optimum Root Set problem, abbreviated ORS, in the context of static analyses expressed in Datalog.

\subsection{Declarative Static Analysis}

Datalog is a logic programming language whose least fixed point semantics makes it suitable to implement static analyses. We say that an analysis is sound when it over-approximates the concrete semantics. We augment the semantics of Datalog in a way that allows us to control the over-approximation.

Figure 4(a) gives the syntax of Datalog: a list of constraints, each constraint having a head on the left and a body on the right. The head is a literal; the body is a list of literals, which we view as a set and can be empty. A literal is a relation applied to a list of arguments, each argument being either a variable or a constant. A tuple is a literal whose arguments are all constants.

Figure 4(b) introduces notations for relations, variables, constants, tuples, and substitutions. It also introduces three special subsets of tuples: alarms, potential causes, and causes. An alarm corresponds to a bug report. A potential cause is a tuple that is identified by a heuristic as possibly spurious. A cause is a tuple that is identified by an oracle (e.g., a human user) as indeed spurious.

Figure 4(c) shows the standard semantics of Datalog. It computes the least fixed point of constraints, which is a set of tuples. Suppose $T$ is the set of derived tuples. Initially, $T$ is empty and we grow it iteratively by instantiating constraints to derive new tuples from existing tuples in $T$. In each iteration, for each rule $l_{0}:-l_{1}, \ldots, l_{n}$, if there exists a substitution $\sigma$ which is a map from variables to constants such that all $\sigma\left(l_{1}\right), \ldots, \sigma(n)$ are in $T$, then we add $\sigma\left(l_{0}\right)$ to $T$. In the first iteration, only constraints with empty bodies are triggered. We use function $S_{C}$ to denote the computation of one iteration. This process continues until $T$ no longer changes. In other words, the Datalog semantics computes the least fixed point of $S_{C}$ (denoted by Ifp $S_{C}$ ). A sound Datalog analysis will derive all tuples that represent valid program facts, but may also derive tuples that represent spurious program facts. 
Figure 4(d) gives the augmented semantics of Datalog, which allows controlling the overapproximation with a set of causes F. It is similar to the standard Datalog semantics except that in each iteration it removes tuples in causes $\mathbf{F}$ from derived tuples $T$ (denoted by function $S F_{C}^{\mathrm{F}}$ ). Since a cause in $\mathbf{F}$ is never derived, it is not used in deriving other tuples. In other words, the augmented semantics do not only suppress the causes but also spurious tuples that can be derived from them.

\subsection{Problem Statement}

We assume the following are fixed: a set $C$ of Datalog constraints, a set $\mathbf{A}$ of alarms, a set $\mathbf{Q}$ of potential causes, and a set $\mathbf{F}$ of confirmed causes. Informally, our goal is to grow $\mathbf{F}$ such that $\llbracket C \rrbracket_{\mathbf{F}}$ contains fewer alarms. To make this precise, we introduce a few terms. Given two sets $T_{1} \supseteq T_{2}$ of tuples, we define the gain of $T_{2}$ relative to $T_{1}$, as the number of alarms that are in $T_{1}$ but not in $T_{2}$ :

$$
\operatorname{gain}\left(T_{1}, T_{2}\right):=\left|\left(T_{1} \backslash T_{2}\right) \cap \mathbf{A}\right|
$$

A root set $\mathrm{R}$ is a non-empty subset of potential but unconfirmed causes: $\mathrm{R} \subseteq \mathrm{Q} \backslash \mathrm{F}$. Intuitively, the name is justified because we will aim to put in $\mathbf{R}$ the root causes of the false alarms. The potential causes in $\mathbf{R}$ are those we will investigate, and for that we have to pay a cost $|\mathbf{R}|$. The potential gain of $\mathbf{R}$ is the gain of $\llbracket C \rrbracket_{\mathrm{F} \cup \mathrm{R}}$ relative to $\llbracket C \rrbracket_{\mathrm{F}}$. The expected payoff of $\mathbf{R}$ is the potential gain per unit cost. (The actual payoff depends on which potential causes in $\mathrm{R}$ will be confirmed.) With these definitions, we can now formally introduce the ORS problem.

Problem 3.1 (Optimum Root $\mathbf{S}$ et). Given a set $C$ of constraints, a set $\mathrm{Q}$ of potential causes, a set $\mathbf{F}$ of confirmed causes, and a set $\mathbf{A}$ of alarms, find a root set $\mathbf{R} \subseteq \mathrm{Q} \backslash \mathbf{F}$ that maximizes $\operatorname{gain}\left(\llbracket C \rrbracket_{\mathrm{F}}, \llbracket C \rrbracket_{\mathrm{F} \cup \mathrm{R}}\right) /|\mathbf{R}|$, which is the expected payoff.

\subsection{Monotonicity}

The definitions from the previous two subsections (Section 3.1 and 3.2) imply certain monotonicity properties. We list these here and refer to them in later sections.

LEMmA 3.2. If $T_{1} \subseteq T_{2}$ and $\mathbf{F}_{1} \supseteq \mathbf{F}_{2}$, then $S F_{C}^{\mathrm{F}_{1}}\left(T_{1}\right) \subseteq S F_{C}^{\mathrm{F}_{2}}\left(T_{2}\right)$. In particular, if $\mathbf{F}_{1} \supseteq \mathbf{F}_{2}$, then $\llbracket C \rrbracket_{\mathrm{F}_{1}} \subseteq \llbracket C \rrbracket_{\mathrm{F}_{2}}$.

Lemma 3.3. If $T_{1} \subseteq T_{1}^{\prime}$ and $T_{2}^{\prime} \subseteq T_{2}$, then gain $\left(T_{1}^{\prime}, T_{2}^{\prime}\right) \geq \operatorname{gain}\left(T_{1}, T_{2}\right)$. Also, gain $(T, T)=0$ for all $T$.

\subsection{NP-Completeness}

Problem 3.1 is an optimization problem which can be turned into a decision problem in the usual way, by providing a threshold for the objective. The question becomes whether there exists a root set $\mathbf{R}$ that can achieve a given gain. This decision problem is clearly in NP: the set $\mathbf{R}$ is a suitable polynomial certificate.

The problem is also NP-hard, which we can show by a reduction from the minimum vertex cover problem. Given a graph $G=(V, E)$, a subset $U \subseteq V$ of vertices is said to be a vertex cover when it intersects all edges $\{i, j\} \in E$. The minimum vertex cover problem, which asks for a vertex cover of minimum size, is well-known to be NP-complete. We reduce it to the ORS problem as follows. We represent the graph $G$ with three relations $\mathbf{R}=$ \{vertex, edge, alarm $\}$. Without loss of generality, let $V$ be the set $\{0, \ldots, n-1\}$, for some $n$. Thus, we identify vertices with Datalog constants. For each edge $\{i, j\} \in E$ we include two ground constraints:

$$
\begin{aligned}
& \operatorname{edge}(i, j):-\operatorname{vertex}(i), \operatorname{vertex}(j) \\
& \operatorname{alarm}():-\operatorname{edge}(i, j)
\end{aligned}
$$


We set $\mathbf{F}=\emptyset, \quad \mathbf{A}:=\{\operatorname{alarm}()\}$, and $\mathbf{Q}:=\{\operatorname{vertex}(i) \mid i \in V\}$. Since $\mathbf{A}$ has size 1 , the gain can only be 0 or 1 . To maximize the ORS objective, the gain has to be 1 . Further, the size of the root set $\mathbf{R}$ has to be minimized. But, one can easily see that a root set leads to a gain of 1 if and only if it corresponds to a vertex cover.

\section{INTERACTIVE ANALYSIS}

Our interactive analysis combines three ingredients: (a) a static analysis; (b) a heuristic; and (c) an oracle. We require the static analysis to be implemented in Datalog, which lets us track dependencies. The requirements on the heuristic and the oracle are mild: given a set of Datalog tuples they must pick some subset. These are all the requirements. Suppose there is a ground truth that returns the truth of each analysis fact based on the concrete semantics. It helps to think of the three ingredients intuitively as follows:

(a) the static analysis is sound, imprecise with respect to the ground truth and fast;

(b) the heuristic is unsound, precise with respect to the ground truth and fast; and

(c) the oracle agrees with the ground truth and is slow.

Technically, we show a soundness preservation result (Theorem 4.4): if the given oracle agrees with the ground truth and the static analysis over-approximates it, then our combined analysis also over-approximates the ground truth. Soundness preservation holds with any heuristic. When a heuristic agrees with the ground truth, we say that it is ideal; when it almost agrees with the ground truth, we say that it is precise. Even though which heuristic one uses does not matter for soundness, we expect that more precise heuristics lead to better performance. We explore speed and precision later, through experiments (Section 6). Also later, we discuss what happens in practice when the static analysis is unsound or the oracle does not agree with the ground truth (Section 4.7).

The high level intuition is as follows. The heuristic is fast. But, since the heuristic might be unsound, we need to consult an oracle before relying on what the heuristic reports. However, consulting the oracle is an expensive operation, so we would like to not do it often. Here is where the static analysis specified in Datalog helps. On one hand, by analyzing the Datalog derivation, we can choose good questions to ask the oracle. On the other hand, after we obtain information from the oracle, we can propagate that information through the Datalog derivation, effectively finding answers to questions we have not yet asked.

We begin by showing how the static analysis, the heuristic, and the oracle fit together (Section 4.1), and why the combination preserves soundness (Section 4.2). A key ingredient in our algorithm is solving the ORS problem. We do this by a sequence of calls to an ILP solver (Section 4.3). Each call to the ILP solver asks whether, given a Datalog derivation, a certain payoff is feasible (Section 4.4 and 4.5). To improve performance, we preprocess the Datalog derivation before constructing the ILP question (Section 4.6). Finally, we close with a short discussion (Section 4.7).

\subsection{Main Algorithm}

Algorithm 1 brings together our key ingredients:

(a) the set $C$ of constraints and the set $\mathrm{A}$ of potential alarms represent the static analysis implemented in Datalog;

(b) Heuristic is the heuristic; and

(c) Decide is the oracle.

The key to combining these ingredients into an analysis that is fast, sound, and precise is the procedure OptimumRootSet, which solves Problem 3.1.

From now on, the set $C$ of constraints and the set $A$ of potential alarms should be thought of as immutable global variables: they do not change, and they will be available in subroutines even 


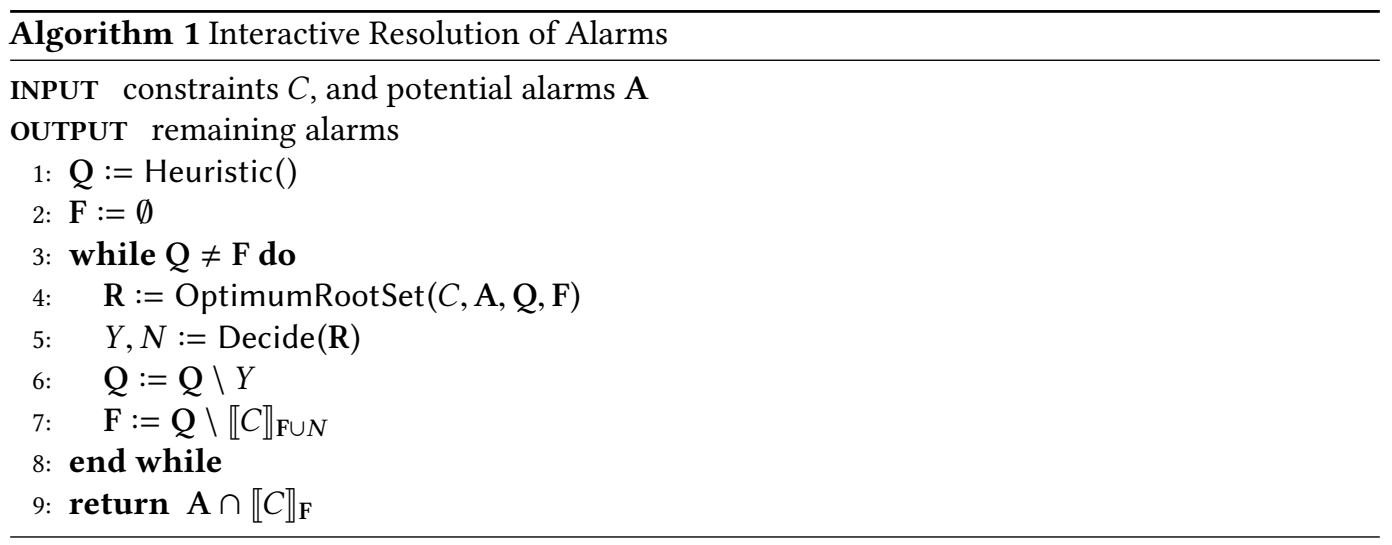

if not explicitly passed as arguments. In contrast, the set $Q$ of potential causes and the set $F$ of confirmed causes do change in each iteration, while maintaining the invariant $\mathbf{F} \subseteq \mathrm{Q}$. Initially, the invariant holds because no cause has been confirmed, $\mathbf{F}=\emptyset$.

In each iteration, we invoke the oracle for a set of potential causes that are not yet confirmed: we call Decide $(\mathbf{R})$ for some non-empty $\mathbf{R} \subseteq \mathbf{Q} \backslash \mathbf{F}$. The result we get back is a partition $(Y, N)$ of $\mathbf{R}$. In $Y$ we have tuples that are in fact true, and therefore are not causes for false alarms; in $N$ we have tuples that are in fact false, and therefore are causes for false alarms. We remove tuples $Y$ from the set $\mathbf{Q}$ of potential causes (line 6); we insert tuples $N$ into the set $\mathbf{F}$ of confirmed causes, and we also insert all other potential causes that may have been blocked by $N$ (line 7).

At the end, we return the remaining alarms $\mathrm{A} \cap \llbracket C \rrbracket_{\mathrm{F}}$.

\subsection{Soundness}

For correctness, we require that the oracle is always right. In particular, the answers given by the oracle should be consistent: if asked repeatedly, the oracle should give the same answer about a tuple. Formally, we require that there exists a partition of all tuples $\mathrm{T}$ into two subsets True and False such that

$$
\operatorname{Decide}(T)=(T \cap \text { True, } T \cap \text { False }) \quad \text { for all } T \subseteq \mathbf{T}
$$

We refer to the set True as the ground truth. We say that the analysis $C$ is sound when

$$
\mathrm{F} \subseteq \text { False implies } \llbracket C \rrbracket_{\mathrm{F}} \supseteq \text { True }
$$

That is, a sound analysis is one that over-approximates, as long as we do not explicitly block a tuple in the ground truth.

Lemma 4.1. A sound analysis $C$ can derive the ground truth; that is, True $=\llbracket C \rrbracket_{\text {False }}$.

The correctness of Algorithm 1 relies on the analysis $C$ being sound and also on making progress in each iteration, which we ensure by requiring

$$
\emptyset \subset \operatorname{OptimumRootSet}(C, \mathrm{~A}, \mathrm{Q}, \mathrm{F}) \subseteq \mathrm{Q} \backslash \mathbf{F}
$$

Lemma 4.2. In Algorithm 1, suppose that Heuristic returns all tuples T. Also, assume (1), (2), and (3). Then, Algorithm 1 returns the true alarms $\mathrm{A} \cap$ True.

Proof Sketch. The key invariant is that

$$
\mathrm{F} \subseteq \text { False } \subseteq \mathrm{Q}
$$


The invariant is established by setting $\mathbf{F}:=\emptyset$ and $\mathrm{Q}:=\mathrm{T}$. By (1), we know that $Y \subseteq$ True and $N \subseteq$ False, on line 5. It follows that the invariant is preserved by removing $Y$ from $\mathrm{Q}$, on line 6. It also follows that $\mathbf{F} \cup N \subseteq$ False and, by (2), that $\llbracket C \rrbracket_{\mathrm{F} \cup N} \supseteq$ True. So, the invariant is also maintained by line 7 . We conclude that (4) is indeed an invariant.

Because $\mathbf{R}$ is non-empty, the loop terminates (details in appendix). When it does, we have $\mathbf{F}=\mathbf{Q}$. Together with the invariant (4), we obtain that $\mathbf{F}=$ False. By Lemma 4.1, it follows that Algorithm 1 returns $\mathrm{A} \cap$ True.

We can relax the requirement that Heuristic returns $\mathrm{T}$ because of the following result:

Lemma 4.3. Consider two heuristics which compute, respectively, the sets $\mathbf{Q}_{1}$ and $\mathbf{Q}_{2}$ of potential causes. Let $A_{1}$ and $A_{2}$ be the corresponding sets of alarms computed by Algorithm 1. If $\mathbf{Q}_{1} \subseteq \mathbf{Q}_{2}$, then $A_{1} \supseteq A_{2}$.

Proof SKeTch. Use the same strategy as in Lemma 4.2's proof, but replace the invariant (4) with

$$
\mathrm{F} \subseteq \mathrm{Q}_{0} \cap \text { False } \subseteq \mathrm{Q}
$$

where $Q_{0} \in\left\{Q_{1}, Q_{2}\right\}$ is the initial value of $Q$.

From Lemmas 4.2 and 4.3, we conclude that Algorithm 1 is sound, for all heuristics.

Theorem 4.4. Assume that there is a ground truth, the analysis $C$ is sound, and OptimumRootSet makes progress; that is, assume (1), (2), and (3). Then, Algorithm 1 terminates and it returns at least the true alarms $\mathrm{A} \cap$ True.

Observe that there is no requirement on Heuristic. If Heuristic always returns $\emptyset$, then our method degenerates to simply using the imprecise but fast static analysis implemented in Datalog. If Heuristic always returns $\mathbf{T}$ and the oracle is a precise analysis, then our method degenerates to an iterative combination between the imprecise and the precise analyses. Usually, we would use a heuristic that has demonstrated its effectiveness in practice, even though it may lack theoretical guarantees for soundness. In our approach, soundness is inherited from the static analysis specified in Datalog and from the oracle. If the oracle is unsound, perhaps because the user makes mistakes, then so is our analysis. Thus, Theorem 4.4 is a relative soundness result.

\subsection{Finding an Optimum Root Set}

We want to find a root set $\mathbf{R}$ that maximizes expected payoff

$$
\frac{\operatorname{gain}\left(\llbracket C \rrbracket_{\mathrm{F}}, \llbracket C \rrbracket_{\mathrm{F} \cup \mathrm{R}}\right)}{|\mathbf{R}|}=\frac{\left|\left(\llbracket C \rrbracket_{\mathrm{F}} \backslash \llbracket C \rrbracket_{\mathrm{F} \cup \mathrm{R}}\right) \cap \mathrm{A}\right|}{|\mathbf{R}|}
$$

This expression is nonlinear, so it is not obvious how to maximize it by using a solver for linear programs. Our solution is to do a binary search on the payoff values, as seen in Algorithm 2. Given a gain $a$, a cost $b$, an analysis of constraints $C$ and alarms $\mathbf{A}$, potential causes $\mathbf{Q}$, and causes $\mathbf{F}$, we find out if a payoff $a / b$ is feasible by calling IsFeasible $(a / b, C, \mathbf{A}, \mathbf{Q}, \mathbf{F})$, which we will implement by invoking an ILP solver (Section 4.5). Similarly, we implement FeasibleSet $(a / b, C, \mathbf{A}, \mathbf{Q}, \mathbf{F})$ using an ILP solver, to find a root set $\mathbf{R} \subseteq \mathbf{Q} \backslash \mathbf{F}$ with payoff $\geq a / b$. We require, as a precondition, that $\mathrm{Q} \backslash \mathrm{F}$ is non-empty. The array ratios contains all possible payoff values, sorted.

\subsection{From Augmented Datalog to ILP}

Let us begin by encoding the augmented Datalog semantics (Figure 4(d)) into ILP: Given F, we want the ILP solver to compute $\llbracket C \rrbracket_{\mathrm{F}}$. Informally, an ILP instance is a set of inequalities and equalities, where variables and constants are constrained to be integers. 


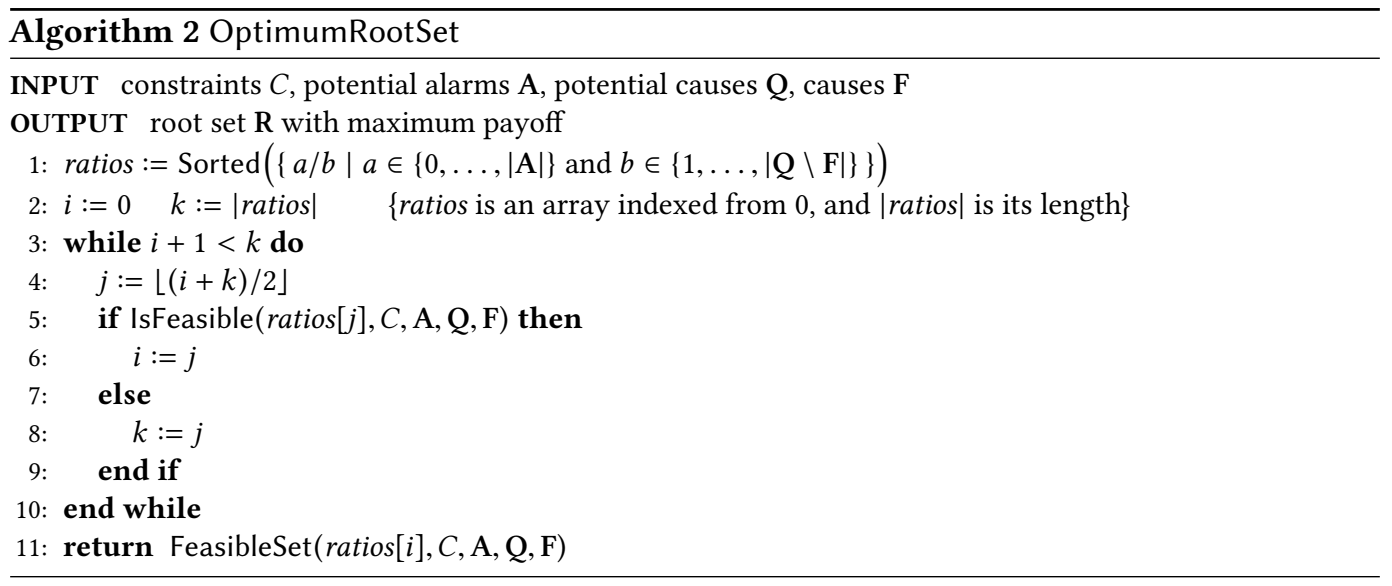

Problem 4.5 (ILP). Given are a matrix $A$ and a vector $b$. Decide whether there exists a non-negative integer vector $x$ such that $A x \geq b$.

The ILP problem is known to be NP-complete [Papadimitriou 1981], as is the ORS problem (Section 3.4). Thus, it is obvious that one problem can be reduced to the other, but we still need a reduction from ORS to ILP which is efficient in practice.

Standard Datalog semantics correspond to the case $\mathbf{F}=\emptyset$. So, to find $\llbracket C \rrbracket_{\emptyset}$ and all rule instances $t_{0}:-t_{1}, \ldots, t_{n}$, we start by running a Datalog solver once. We set $\mathbf{T}:=\llbracket C \rrbracket_{\emptyset}$. Knowing the result of the standard Datalog solver, the task is to construct an ILP instance that would compute $\llbracket C \rrbracket_{\mathrm{F}}$ for an arbitrary $\mathbf{F}$. For each tuple $t$, we introduce variables $x_{t}, y_{t}, z_{t}$ with values in $\{0,1\}$. Let $X$ be the set $\left\{t \in \mathrm{T} \mid x_{t}=1\right\}$, and define $Y$ and $Z$ similarly. We will construct our ILP instance such that $X=\llbracket C \rrbracket_{\mathrm{F}}, Y=S_{C}(X)$, and $Z=\mathrm{F}$. We encode $Z=\mathrm{F}$ by having a constraint

$$
z_{t}=\left\{\begin{array}{ll}
0 & \text { if } t \notin \mathbf{F} \\
1 & \text { if } t \in \mathbf{F}
\end{array} \quad \text { for each } t \in \mathbf{T}\right.
$$

We encode $Y \supseteq S_{C}(X)$ by having constraints

$$
y_{t_{0}}+\sum_{k=1}^{n}\left(1-x_{t_{k}}\right)>0
$$

for each $t_{0}:-t_{1}, \ldots, t_{n}$

Finally, we encode $X \supseteq Y \backslash Z$ by

$$
x_{t}+\left(1-y_{t}\right)+z_{t}>0 \quad \text { for each } t \in \mathbf{T}
$$

Observe that $X \supseteq Y \backslash Z$ together with $Y \supseteq S_{C}(X)$ imply that $X \supseteq S_{C}^{Z}(X)$; that is, $X$ is a post fixed point of $S F_{C}^{Z}$. By Lemma 3.2 and the Knaster-Tarski theorem, the least post fixed point of $S F_{C}^{Z}$ coincides with its least fixed point. Thus, to guarantee that $X=\llbracket C \rrbracket_{\mathrm{F}}$, it only remains to add the optimization objective to minimize $\sum_{t \in \mathrm{T}} x_{t}$. Note $X=\llbracket C \rrbracket_{\mathrm{F}}$ implies $X=S F_{C}^{Z}(X)$, which together with $X \supseteq Y \backslash Z \supseteq S F_{C}^{Z}(X)$ imply that $Y=S_{C}(X)$.

Remark 4.6. The constraints we used are not immediately of the form required by Problem 4.5, but they can be rewritten easily. Moreover, Problem 4.5 does not have an optimization objective, but this is, again, a minor concern: in practice, most solvers allow optimization objectives; in theory, one can always do binary search. 


$$
\begin{aligned}
y_{t_{0}}+\sum_{k=1}^{n}\left(1-x_{t_{k}}\right) & >0 & & \text { for all } t_{0}:-t_{1}, \ldots, t_{n} \\
x_{t}+\left(1-y_{t}\right)+z_{t} & >0 & & \text { for } t \in \mathbf{T} \\
z_{t} & =0 & & \text { for } t \notin \mathbf{Q} \\
z_{t} & =1 & & \text { for } t \in \mathbf{F} \\
\sum_{t \in \mathbf{Q} \backslash \mathbf{F}} z_{t} & >0 & & \text { (requires }|\mathbf{R}|>0) \\
b \sum_{t \in A \cap \llbracket C \prod_{\mathbf{F}}}\left(1-x_{t}\right)-a \sum_{t \in \mathbf{Q} \backslash \mathbf{F}} z_{t} & \geq 0 & & \text { (requires payoff } \geq a / b)
\end{aligned}
$$

Fig. 5. Implementing IsFeasible and FeasibleSet as an ILP instance. All variables $x_{t}, y_{t}, z_{t}$ take values in $\{0,1\}$.

We use the ILP solver as follows. Given a set F, we create constraints as above. Then we run the ILP solver, which will return a variable assignment. The desired $\llbracket C \rrbracket_{\mathrm{F}}$ is encoded in the assignment of the variables $\left\{x_{t}\right\}_{t \in \mathrm{T}}$.

\subsection{Feasible Payoffs}

In this section, we adjust the ILP encoding of augmented Datalog so that we can implement the subroutines IsFeasible and FeasibleSet used in Algorithm 2. Given sets A, Q, F and a rational payoff $a / b$, we want to decide whether there exists a non-empty root set $\mathbf{R} \subseteq \mathbf{Q} \backslash \mathbf{F}$ that achieves the payoff (IsFeasible), and find an example of such a set (FeasibleSet). In other words, we want to find a set $\mathbf{R}$ such that

$$
b \cdot\left|\left(\llbracket C \rrbracket_{\mathrm{F}} \backslash \llbracket C \rrbracket_{\mathrm{F} \cup \mathrm{R}}\right) \cap \mathrm{A}\right|-a \cdot|\mathbf{R}| \geq 0
$$

and $|\mathbf{R}|>0$. The resulting encoding appears in Figure 5. As before, we use variables $x_{t}, y_{t}, z_{t}$, but with slightly different meanings: The constraints are set up such that $X \supseteq \llbracket C \rrbracket_{Z}$ and $Z=\mathbf{F} \cup \mathbf{R}$. As before (Section 4.4), constraints (1)-(4) in Figure 5 encode $Y \supseteq S_{C}(X)$ and $X \supseteq Y \backslash Z$, which imply that $X$ is a post fixed point of $S F_{C}^{Z}$. We dropped the optimization objective that ensured we compute least fixed points, which is why $X \supseteq \llbracket C \rrbracket_{Z}$ rather than $X=\llbracket C \rrbracket_{Z}$. However, using Lemma 3.3, we can show that there exists a post fixed point of $S F_{C}^{Z}$ that leads to the payoff $a / b$ (that is, satisfies constraints (5) and (6) in Figure 5) if and only if the least fixed point of $S F_{C}^{Z}$ leads to the payoff. So, the minimization of $\sum_{t \in \mathrm{T}} x_{t}$ is unnecessary.

Example 4.7. We show the IsFeasible encoding for the second iteration of the example from Section 2. We shorten each relation name to its first letter.

We first show the encoding that corresponds to constraints (1) in Figure 5. Recall the derivation graph from Figure 3. From the rule instances with head s, we obtain the following ILP constraints:

$$
y_{t}+\left(1-x_{\mathrm{e}(25)}\right)>0 \quad \text { for } t \in\{\mathrm{s}(46), \mathrm{s}(57), \mathrm{s}(71), \mathrm{s}(72), \ldots\}
$$

From the rule instances with head $r$, we obtain

$$
y_{\mathrm{r}(46,57)}+\left(1-x_{\mathrm{s}(46)}\right)+\left(1-x_{\mathrm{s}(57)}\right)>0
$$

and eight other similar constraints. The input e(25) is modeled by a rule with an empty body, so $y_{\mathrm{e}(25)}>0$.

Then, for every tuple $t$ in Figure 3, we add $x_{t}+\left(1-y_{t}\right)+z_{t}>0$, which correspond to constrains (2) in Figure 5. 
The potential causes returned by the heuristic are $Q=\{s(46), s(47), s(48)\}$. At the beginning of the second iteration one cause had been confirmed, $\mathbf{F}=\{\mathrm{s}(47)\}$. We have $z_{t}=1$ for $t \in \mathrm{F}$, and $z_{t}=0$ for $t \notin \mathrm{Q}$, which correspond to constraints (4) and (3) in Figure 5 respectively.

For constraints (5), we have $z_{\mathrm{s}(46)}+z_{\mathrm{s}(48)}>0$.

Finally,

$$
b \cdot\left(1-x_{\mathrm{r}(46,57)}+1-x_{\mathrm{r}(46,71)}+1-x_{\mathrm{r}(46,72)}+1-x_{\mathrm{r}(48,74)}\right)-a \cdot\left(z_{\mathrm{s}(46)}+z_{\mathrm{s}(48)}\right) \geq 0
$$

where the indices of $x$ range over the four unresolved alarms at the start of the second iteration.

Let us see what we need to write down the constraints from Figure 5. First, we need the result of running a standard Datalog solver: the set $\llbracket C \rrbracket_{\emptyset}$, and the corresponding rule instances $t_{0}:-t_{1}, \ldots, t_{n}$. We obtain these by running the Datalog solver once, in the beginning. Second, we need the sets A, $\mathrm{Q}$, and $\mathrm{F}$. The set $\mathbf{A}$ is fixed, since it only depends on the analysis. Sets $\mathbf{Q}$ and $\mathbf{F}$ do change, but they are sent as arguments to IsFeasible and FeasibleSet. Third, we need the ratio $a / b$, which is also sent as an argument. Fourth, we need the set $\llbracket C \rrbracket_{F}$; we can compute it as described earlier (Section 4.4).

If the ILP solver finds the instance to be feasible, then it gives us an assignment for variables, including for $\left\{z_{t}\right\}_{t \in \mathbf{T}}$. To implement FeasibleSet, we compute $\mathbf{R}$ as $Z \backslash \mathbf{F}$.

\subsection{Preprocessing}

Using the encoding presented so far, we can solve the ORS problem by invoking an ILP solver. However, sometimes the ILP solver would take tens of minutes to reply. In this section, we see two preprocessing techniques that, together, empirically reduce the time spent in the ILP solver by a factor of 62 on average.

Partial Evaluation. We evaluate $\llbracket C \rrbracket_{\mathrm{F}}$ several times, for the same set $C$ of constraints but for different sets $\mathbf{F}$. Since we are always interested in potential causes, $\mathbf{F} \subseteq \mathbf{Q}$, we know by Lemma 3.2 that $\llbracket C \rrbracket_{\mathrm{F}} \supseteq \llbracket C \rrbracket_{\mathrm{Q}}$. It is thus beneficial to precompute $\llbracket C \rrbracket_{\mathrm{Q}}$. Then, for each tuple $t \in \llbracket C \rrbracket_{\mathrm{Q}}$, we remove the constraints that have head $t$, and then we remove $t$ from the remaining constraints. The resulting set of constraints, which is much smaller, is used for all future calls to the ILP solver.

Tuple Elimination. Since we are not interested in tuples that are intermediate between potential causes and potential alarms, we can sometimes take shortcuts. Suppose a tuple $t \notin Q \cup A$ appears in only two constraints: $t^{\prime \prime}:-t$ and $t:-t^{\prime}$. Then, we can eliminate $t$ by replacing the two constraints with one: $t^{\prime \prime}:-t^{\prime}$. More generally, if all occurrences of $t$ are in the $m+n$ constraints

$$
t_{1}^{\prime \prime}:-t \quad t_{2}^{\prime \prime}:-t \quad \ldots \quad t_{m}^{\prime \prime}:-t \quad \text { and } \quad t:-T_{1}^{\prime} \quad t:-T_{2}^{\prime} \quad \ldots \quad t:-T_{n}^{\prime}
$$

then we can replace all these with the $m n$ constraints

$$
t_{i}^{\prime \prime}:-T_{j}^{\prime} \quad \text { for all } i \in\{1, \ldots, m\} \text { and } j \in\{1, \ldots, n\}
$$

It is profitable to do so when $m n \leq m+n$.

These preprocessing techniques are simple but effective. They are both inspired by techniques used in SAT solving: Partial evaluation is similar to unit propagation, and tuple elimination is similar to variable elimination. We cannot simply use a SAT solver, though, for several reasons. First, constraints like (6) in Figure 5 are cumbersome to express as Boolean formulas because of the integers $a$ and $b$. Second, although SAT solvers usually implement variable elimination, MaxSAT solvers do not. We would need a MaxSAT solver because we want to find a small R (Section 4.7). And third, note that tuple elimination uses domain specific knowledge: which tuples are potential causes or potential alarms. Still, our main contribution here is to observe that applying these known techniques is crucial if one wants to use an off-the-shelf ILP solver on instances arising from Datalog-based static analysis. 


\subsection{Discussion}

First, we discuss a few alternatives for the payoff, for the termination condition, and for the oracle. Then, we discuss the issue of soundness from the point of view of the mismatch between theory and practice. Finally, we discuss how our approach could be applied in a non-Datalog setting.

Payoff. The algorithm presented above uses $|\mathbf{R}|$ as the cost measure. It might be, however, that some potential causes are more difficult to investigate than others. If the user provides us with an expected cost of investigating each potential cause, then we can adapt our algorithm, in the obvious way, so that it prefers calling Decide on cheap potential causes.

In situations when multiple root sets have the same maximum payoff, we may want to prefer one with minimum size. Intuitively, small root sets let us gather information from the oracle quickly. To encode a preference for smaller root sets, we can extend the constraints in Figure 5 with the optimization objective to minimize $\sum_{t \in \mathrm{Q} \backslash \mathrm{F}} z_{t}$.

Termination. Algorithm 1 terminates when all potential causes have been investigated; that is, when $Q=F$. Another option is to look at the current value of the expected payoff. Suppose Algorithm 2 finds an expected payoff $\leq 1$. This means that we expect that it will not be easier to investigate potential causes rather than investigate the alarms themselves. So, we could decide to terminate, as we do in our experiments (Section 6). Finally, instead of looking at the expected payoff computed by the ILP solver, we could track the actual payoff for each iteration. Then, we could stop if the average payoff in the last few iterations is less than some threshold.

Oracle. By default, the oracle Decide is a human user. However, we can also use a precise yet expensive analysis as the oracle, which enables an alternative use case of our approach. This use case focuses on balancing the overall precision and scalability of combining the base analysis and the oracle analysis, rather than reducing user effort in resolving alarms. Our approach allows the oracle analysis to focus on only the potential causes that are relevant to the alarms, especially the ones with high expected payoffs. For example, the end user might find it too long a time to apply the oracle analysis to resolve all potential causes. By applying our approach, they can use the oracle analysis to only answer the potential causes with high payoffs and resolve the rest alarms via other methods (e.g., manual inspection). Appendix B includes a more detailed discussion of this use case.

Soundness. In practice, most static analyses are unsound [Livshits et al. 2015]. In addition, in our setting, the user may answer incorrectly. We discuss each of these issues below.

Many program analyses are designed to be sound in theory but are unsound in practice due to engineering compromises. If certain language features were handled in a sound manner, then the analysis would be unscalable or exceedingly imprecise. For example, in Java, such features include reflection, dynamic class loading, native code, and exceptions. If we start from an unsound static analysis, then our interactive analysis is also unsound. However, Theorem 4.4 gives evidence that we do not introduce new sources of unsoundness. More importantly, our approach is still effective in suppressing false alarms and therefore reduces user effort, as we demonstrate through experiments (Section 6).

In theory, the oracle never makes mistakes; in practice, users do make mistakes, of two kinds: they may label a true tuple as spurious, and they may label a spurious tuple as true. If a true tuple is labeled as spurious, then the interactive analysis becomes unsound. However, if the user answers $x \%$ of questions incorrectly, then we expect that the fraction of false negatives is not far from $x \%$. Our experiments show that, even better, the fraction of false negatives tends to be less than $x \%$. A consequence of this observation is that, if potential causes are easier to inspect than alarms, then our approach will decrease the chances that real bugs are missed. 
If a spurious tuple is labeled as true, then the interactive analysis may ask more questions. It is also possible that fewer false alarms are filtered out: the user could make the mistake on the only remaining question with expected payoff $>1$, which in turn would cause the interaction to terminate earlier. (See the previous discussion on termination.)

Later, we analyze both kinds of mistakes quantitatively (Section 6.2 and Table 2).

Non-Datalog Analyses. We focus on program analyses implemented in Datalog for two reasons: (1) it is easy to capture provenance information for such analyses; and (2) there is a growing trend towards specifying program analyses in Datalog [Jordan et al. 2016; Madsen et al. 2016; Mangal et al. 2015; Smaragdakis and Bravenboer 2010; Zhang et al. 2014]. However, not all analyses are implemented in Datalog; for example, see Ayewah et al. [2008]; Bessey et al. [2010]; Copeland [2005]. In principle, it is possible to apply our approach to any program analysis. To do so, the analysis designer would need to figure out how to capture provenance information of an analysis' execution. While this might be a cumbersome task, however, it constitutes a one-time effort.

\section{INSTANCE ANALYSES}

We demonstrate our approach on the static datarace analysis in Chord for Java programs. To show the generality and versatility of our approach, Appendix B describes its instantiation on a pointer analysis for the alternative use case, where the oracle is a precise but expensive static analysis. Next, we briefly describe the datarace analysis, its notions of alarms and causes, and our implementation of the procedure Heuristic.

The datarace analysis is a context- and flow-sensitive analysis introduced by Naik et al. [2006]. It comprises 30 rules, 18 input relations, and 18 output relations. It combines a thread-escape analysis, a may-happen-in-parallel analysis, and a lockset analysis. It reports a datarace between each pair of instructions that access the same thread-shared object, are reachable by different threads in parallel, and are not guarded by a common lock.

While the alarms are the datarace reports, the potential causes, which are identified by Heuristic, could be any set of tuples in theory. However, we found it useful to focus our heuristics on two relations: shared and parallel, which we observe to often contain common root causes of false alarms. The shared relation contains instructions that may access thread-shared objects; the parallel relation contains instruction pairs that may be reachable in parallel. We call the set of all tuples from these two relations the universe of potential causes.

We provide four different Heuristic instantiations, which are shown in Figure 6. Instantiations static_optimistic and static_pessimistic contain static rules that reflect analysis designers' intuition. Instantiation dynamic leverages a dynamic analysis to identify analysis facts that are likely spurious. Finally, instantiation aggregated combines the previous three instantiations using a decision tree. We next describe each instantiation in detail.

Instantiation static_optimistic encodes a heuristic applied in the implementation by Naik et al. [2006], which treats shared tuples whose associated accesses occur in an object constructor as spurious. Moreover, it includes parallel tuples related to instructions in Thread.run() and Runnable.run() in the potential causes as they are often falsely derived due to a context-insensitive call-graph. Instantiation static_pessimistic is similar to static_optimistic except it aggressively classifies all shared tuples as false, capturing the intuition that most accesses are thread-local.

When applying our approach, one might lack intuitions like the above ones to effectively identify potential causes. In this case, they can leverage the power of testing, akin to work on discovering likely invariants [Ernst et al. 2001]: if an analysis fact is not observed consistently across different runs, then it is very likely to be false. We provide instantiation dynamic to capture this intuition. It 


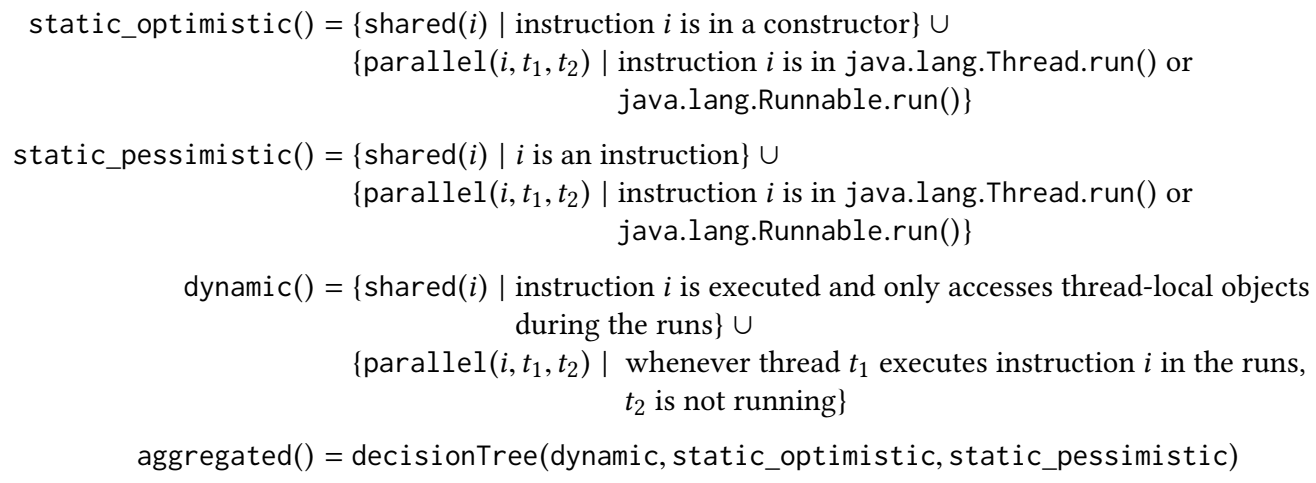

Fig. 6. Heuristic instantiations for the datarace analysis.

Table 1. Benchmark characteristics. Column $|\mathrm{A}|$ shows the numbers of alarms. Column $\left|\mathbf{Q}_{U}\right|$ shows the sizes of the universes of potential causes, where $k$ stands for thousands. All the reported numbers except for $|\mathrm{A}|$ and $\left|Q_{U}\right|$ are computed using a 0 -CFA call-graph analysis.

\begin{tabular}{|c|c|c|c|c|c|c|c|c|c|c|c|c|c|}
\hline & \multirow{2}{*}{ Description } & \multicolumn{2}{|c|}{ \# Classes } & \multicolumn{2}{|c|}{ \# Methods } & \multicolumn{2}{|c|}{ Bytecode (KB) } & \multicolumn{2}{|c|}{ Source (KLOC) } & \multicolumn{3}{|c|}{$|\mathrm{A}|$} & \multirow[t]{2}{*}{$\left|Q_{U}\right|$} \\
\hline & & app & total & app & total & app & total & app & total & false & total & false $\%$ & \\
\hline raytracer & $3 \mathrm{D}$ raytracer & 18 & 87 & 74 & 283 & 5.1 & 18 & 1.8 & 41.4 & 226 & 411 & $55 \%$ & $5.3 \mathrm{k}$ \\
\hline montecarlo & financial simulator & 18 & 114 & 115 & 442 & 5.2 & 23 & 3.5 & 50.8 & 37 & 38 & $97.4 \%$ & $4.4 \mathrm{k}$ \\
\hline sor & successive over relaxation & 6 & 100 & 12 & 431 & 1.8 & 30 & 0.6 & 52.5 & 64 & 64 & $100 \%$ & 940 \\
\hline elevator & discrete event simulator & 5 & 188 & 24 & 899 & 2.3 & 52 & 0.6 & 88 & 100 & 100 & $100 \%$ & $1.4 \mathrm{k}$ \\
\hline jspider & web spider engine & 113 & 391 & 422 & 1572 & 17.7 & 74.6 & 6.7 & 106 & 214 & 264 & $81.1 \%$ & $82 \mathrm{k}$ \\
\hline hedc & web crawler from ETH & 44 & 353 & 230 & 2,134 & 16 & 140 & 6.1 & 128 & 317 & 378 & $83.9 \%$ & $38 \mathrm{k}$ \\
\hline ftp & Apache FTP server & 119 & 527 & 608 & 2,705 & 36.5 & 142 & 18.2 & 162 & 594 & 787 & $75.5 \%$ & $131 \mathrm{k}$ \\
\hline weblech & website download/mirror tool & 11 & 576 & 78 & 3,326 & 6 & 208 & 12 & 194 & 6 & 13 & $46.2 \%$ & $6.2 \mathrm{k}$ \\
\hline
\end{tabular}

leverages a dynamic analysis and returns tuples whose associated program points are reached but associated program facts are not observed across runs.

Having multiple Heuristic instantiations is not unusual; we gain the benefits of each of them using a combined instantiation aggregated, which decides whether to classify each given tuple as a potential cause by considering the results of all the individual instantiations. Instantiation aggregated realizes this idea by using a decision tree that aggregates the results of the other instantiations. We obtain such a decision tree by training it on benchmarks where the tuples in the universe of potential causes are fully labeled.

\section{EMPIRICAL EVALUATION}

This section evaluates the effectiveness of our approach by applying it to the datarace analysis on a suite of 8 Java programs. In addition, Appendix B discusses the evaluation results for the use case where the oracle is a precise yet expensive analysis, by applying our approach to the pointer analysis on the same benchmark suite.

\subsection{Evaluation Setup}

We implemented our approach in a tool called URSA for analyses specified in Datalog that target Java programs. We use Chord [Naik 2006] as the Java analysis framework, bddbddb [Whaley et al. 2005] as the Datalog solver, and Gurobi [Gurobi Optimization, Inc. 2016] as the ILP solver. All experiments were done using Oracle HotSpot JVM 1.6 on a Linux machine with 64GB memory and $2.7 \mathrm{GHz}$ processors. 
Table 1 shows the characteristics of each benchmark. In particular, it shows the numbers of alarms and tuples in the universes of potential causes. Note that the size of the universe of potential causes is one to two orders of magnitude bigger than the number of alarms for each benchmark, highlighting the large search space of our problem.

We next describe how we instantiate the main algorithm (Algorithm 1) of URSA by describing our implementations of Heuristic, Decide, and the termination check.

Heuristic. We evaluate all Heuristic instantiations described in Section 5, plus an ideal one that answers according to the ground truth. We define aggregated by

aggregated() $:=\left\{t \in Q_{U} \mid f(t \in\right.$ static_optimistic ()$, t \in$ static_pessimistic ()$, t \in$ dynamic ()$\left.)\right\}$

where $f$ is a ternary Boolean function represented by a decision tree. We learn this decision tree, using the C4.5 algorithm [Quinlan 1993], on the four smaller benchmarks. Each data point corresponds to a tuple in the universes of potential causes and we obtain the expected output by invoking the Decide procedure, whose implementation is described immediately after the current discussion on Heuristic. The result of learning is $f(x, y, z)=x \wedge z$, which leads to

$$
\text { aggregated }()=\text { static_optimistic }() \cap \text { dynamic }() \text {. }
$$

Thus, aggregated only classifies a tuple as spurious when both static_optimistic and dynamic do so. We find aggregated to be the best instantiation overall in terms of numbers of questions asked and alarms resolved by URsA.

To evaluate the effectiveness of various heuristics, we also define the heuristic ideal() := $Q_{U} \cap$ True. This heuristic requires precomputing the ground truth, which one would not do for using URsA normally. As ground truth, we use consensus between answers from many users. Note that the interactive analysis will treat ideal as it does with any of the other heuristics, cross-checking its answers against the oracle.

Decide. In practice, Decide is implemented by a real user or an expensive analysis that provides answers to questions posed by URSA in an online manner. To evaluate our approach uniformly under various settings, however, we obtained answers to all the alarms and potential causes offline. To obtain such answers, we hired a group of 44 Java developers on UpWork [upw 2015], a freelancer platform. For improved confidence in the answers, we required them to have prior experience with concurrent programming in Java; moreover, we filtered out 4 users who gave incorrect answers to three hidden diagnostic questions, resulting in 40 valid participants. Finally, to reduce the answering effort, we applied a dynamic analysis and marked facts observed to hold in concrete runs as true, and required answers only for the unresolved ones.

Termination. As described in Section 4.7, we decide to terminate the algorithm when the expected payoff is $\leq 1$. Intuitively, there is no root cause left that could explain more than one alarm. Thus, the user may find it more effective to inspect the remaining reports directly or use other techniques to further reduce the false alarms.

\subsection{Evaluation Results}

Our evaluation addresses the following five questions:

(1) Generalization: How effectively does URsA identify the fewest queries that eliminate the most false alarms?

(2) Prioritization: How effectively does Ursa prioritize those queries that eliminate the most false alarms?

(3) User time for causes vs. alarms: How much time do users spend inspecting a potential cause versus a potential alarm? 


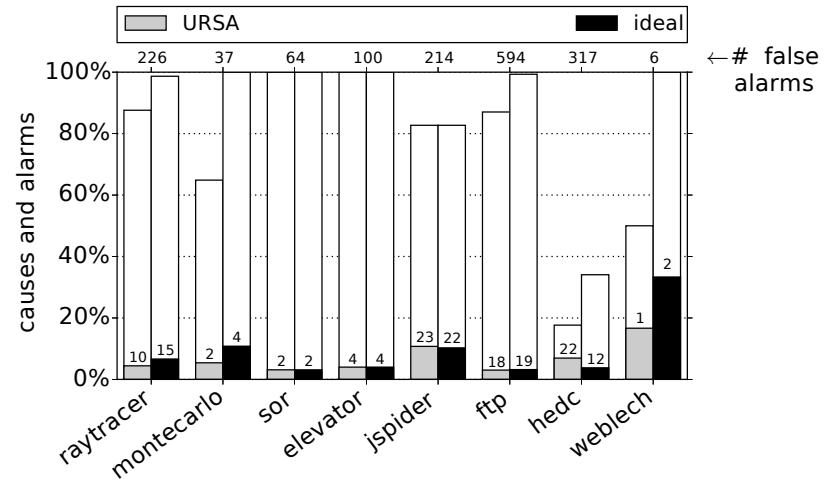

Fig. 7. Number of questions asked over total number of false alarms (denoted by the lower dark bars) and percentage of false alarms resolved (denoted by the upper light bars) by URSA. Note that URSA terminates when the expected payoff is $\leq 1$, which indicates that the user should stop looking at potential causes and focus on the remaining alarms.
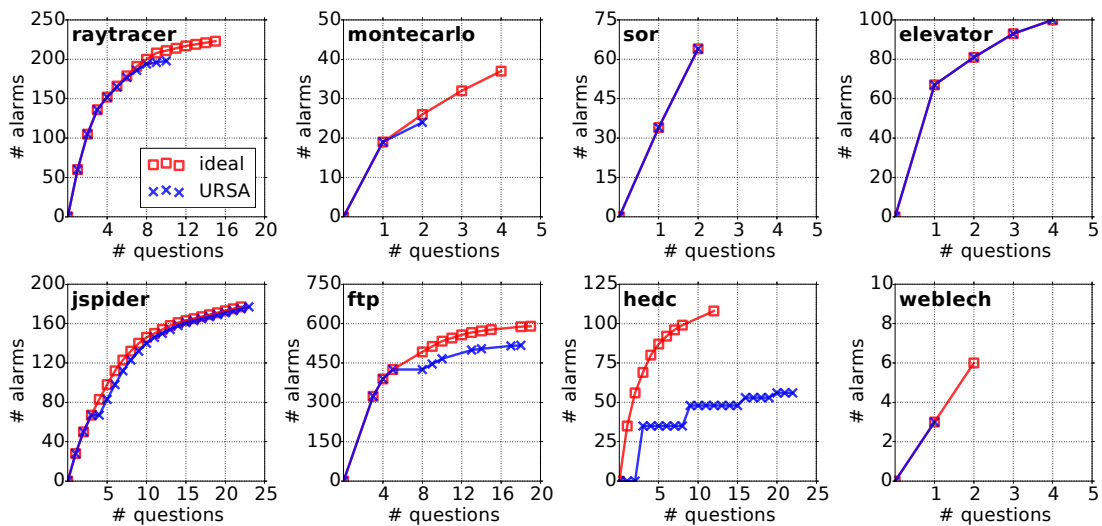

Fig. 8. Number of questions asked and number of false alarms resolved by URSA in each iteration.

(4) Impact of incorrect user answers: How do incorrect user answers affect the effectiveness of URSA in terms of precision, soundness, and user effort?

(5) Scalability: Does the optimization procedure of URSA scale to large programs?

(6) Effect of different heuristics: What is the impact of different heuristics on generalization and prioritization?

We report all averages using arithmetic means except average payoffs and average speedups, which are calculated using geometric means.

Generalization results. Figure 7 shows the generalization results of URSA with aggregated, the best available Heuristic instantiation. For comparison, we also include the ideal heuristic. For each benchmark under both settings, we show the percentage of resolved false alarms (the upper light bars) and the number of asked questions over the total number of false alarms (the lower dark bars). The figure also shows the absolute numbers of asked questions (denoted by the numbers over dark bars) and the numbers of false alarms produced by the input static analysis (denoted by the numbers at the top).

URSA is able to eliminate $73.7 \%$ of the false alarms with an average payoff of $12 \times$ per question. On $\mathrm{ftp}$, the benchmark with the most false alarms, the gain is as high as $87 \%$ and the average payoff 
increases to $29 \times$. Note that URSA does not resolve all alarms as it terminates when the expected payoff becomes 1 or smaller, which means there are no common root causes for the remaining alarms. These results show that most of the false alarms can indeed be eliminated by inspecting only a few common root causes.

URSA eliminates most of the false alarms for all benchmarks except hedc, where only $17.7 \%$ of the false alarms are eliminated. In fact, even under the ideal setting, only $34 \%$ of the false alarms can be eliminated. Closer inspection revealed that most alarms in hedc indeed do not share common root causes. However, the questions asked comprise only $7 \%$ of the false alarms. This shows that even when there is scarce room to generalize, URSA does not ask unnecessary questions.

In the ideal case, URSA eliminates an additional $15.6 \%$ of false alarms on average, which modestly improves over the results with aggregated. We thus conclude the aggregated instantiation is effective in identifying common root causes of false alarms.

Prioritization results. Figure 8 shows the prioritization results of UrsA. In the plots, each iteration of Algorithm 1 has a corresponding point, which represents the number of false alarms eliminated (y-axis) and the number of questions (x-axis) asked so far. As before, we compare the results of URSA to the ideal case, which has a perfect heuristic.

We observe that a few causes often yield most of the benefits. For instance, three causes can resolve 323 out of 594 false alarms on ftp, yielding a payoff of $108 \times$. URsA successfully identifies these causes with high payoffs and poses them to the user in the earliest few iterations. In fact, for the first three iterations on most benchmarks, URSA asks exactly the same set of questions as the ideal setting. The results of these two settings only differ in later iterations, where the payoff becomes relatively low. We also notice that there can be multiple causes in the set that gives the highest benefit (for instance, the aforementioned $\mathrm{ftp}$ results). The reason is that there can be multiple derivations to each alarm. If we naively search the potential causes by fixing the number of questions in advance, we can miss such causes. URSA, on the other hand, successfully finds them by solving the optimal root set problem iteratively.

The fact that URSA is able to prioritize causes with high payoffs allows the user to stop interacting after a few iterations and still get most of the benefits. URSA terminates either when the expected payoff drops to 1 or when there are no more questions to ask. But in practice, the user might choose to stop the interaction even earlier. For instance, the user might be satisfied with the current result or she may find limited payoff in answering more questions.

We study these causes with high payoffs more closely. For our datarace analysis, the two main categories of causes are (i) spurious thread-shared memory access (shared) tuples in object constructors of classes that extend the java. lang. Thread class or implement the java. lang. Runnable interface, and (ii) spurious may-happen-in-parallel (parallel) tuples in the run methods of similar classes. The objects whose constructors contain spurious (shared) tuples are mostly created in loops where a new thread is created and executes the run methods of these objects in each iteration. The datarace analysis is unable to distinguish the objects created in different iterations and considers them all as thread-shared after the first iteration. This leads to many false datarace alarms between the main thread which invokes the constructors and the threads created in the loops which also access the objects by executing their run methods. The spurious parallel tuples are produced due to the context-insensitive call-graphs which mismatch the run methods containing them to Thread. start invocations that cannot actually reach these run methods. This in turn leads to many false alarms between multiple threads.

User time for causes vs. alarms. While URSA can significantly reduce the number of alarms that a user must inspect, it comes at the expense of the user inspecting causes. We measured the time consumed by each programmer when labeling individual causes and alarms for the datarace

Proc. ACM Program. Lang., Vol. 1, No. OOPSLA, Article 57. Publication date: October 2017. 
Table 2. Results of URsA on ftp with noise in Decide. The baseline analysis produces 193 true alarms and 594 false alarms. We run each setting for 30 times and take the averages.

\begin{tabular}{r|r|r|r|r|r|r}
\hline$\%$ Noise & $\begin{array}{c}\text { \# Resolved } \\
\text { False Alarms }\end{array}$ & $\begin{array}{c}\text { \% Resolved } \\
\text { False Alarms }\end{array}$ & $\begin{array}{c}\text { \# False } \\
\text { Negatives }\end{array}$ & $\begin{array}{c}\text { \% Retained } \\
\text { True Alarms }\end{array}$ & \# Questions & Payoff \\
\hline $0 \%$ & 517.0 & $87.0 \%$ & 0.0 & $100.0 \%$ & 18.0 & 28.7 \\
$1 \%$ & 516.4 & $86.9 \%$ & 0.0 & $100.0 \%$ & 18.1 & 28.6 \\
$5 \%$ & 515.4 & $86.8 \%$ & 4.9 & $97.4 \%$ & 18.2 & 28.4 \\
$10 \%$ & 505.0 & $85.0 \%$ & 9.2 & $95.2 \%$ & 19.4 & 26.3 \\
\hline
\end{tabular}

analysis. Our measurements show that it takes 578 seconds on average for a user to inspect a datarace alarm but only 265 seconds on average to inspect a cause. This is because reasoning about an alarm often requires reasoning about multiple causes and other program facts that can derive it. To precisely quantify the reduction in user effort, a more rigorous user study is needed, which is beyond the scope of the current paper. However, the massive reduction in the number of alarms that a user needs to inspect and the fact that a cause is on average much less expensive to inspect shows URSA's potential to significantly reduce overall user effort.

In practice, there might be cases where the causes are much more expensive to inspect than alarms. However, as discussed in Section 4.7, as long as the costs can be quantified in some way, we can naturally encode them in our optimization problem. As a result, URSA will be able to find the set of questions that maximizes the payoff in user effort.

Impact of incorrect user answers. As we discussed earlier (Section 4.7), users can make mistakes. We analyze the impact of mistakes quantitatively by injecting random noise in Decide: we flip its answer to each tuple with probability $x \%$. We set $x$ to $1,5,10$, and apply URSA on $\mathrm{ftp}$, the benchmark with the most alarms. We run the experiment under each setting for 30 times and calculate the averages of all statistics. Table 2 summarizes the results in terms of precision, soundness, and user effort. We also show the results of URSA without noise as a comparison.

Columns 2 and 3 show, respectively, the number and the percentage of false alarms that are resolved by URSA with noise. When the amount of noise increases, both statistics drop. This is due to Decide incorrectly marking certain spurious tuples posed by URSA as true. These tuples might well be the only tuples that can resolve certain alarms and are with payoff $>1$. However, we also notice that the drop is modest: a noise of $10 \%$ leads to a drop in resolved false alarms of $2 \%$ only.

Columns 4 and 5 show, respectively, the number of false negatives and the percentage of retained true alarms. URSA can introduce false negatives when a noisy Decide incorrectly marks a true tuple as spurious. The number of false negatives grows as the amount of noise increases, but the growth is not significant: a noise of $10 \%$ leads to missing $4.8 \%$ true alarms only. This shows that URSA does not amplify user errors. It is especially true for datarace analysis, given that its alarms are harder to inspect compared to the root causes.

Columns 6 and 7 show, respectively, the number of questions asked by URSA and the payoff of answering these questions. As expected, more noise leads to more questions and smaller payoffs. But, the effect is modest: a noise of $10 \%$ increases the number of questions by $7.7 \%$ and decreases the payoff by $8.4 \%$.

In summary, URSA is resilient to user mistakes.

Scalability results. Figure 9 shows the distributions of the time consumed by Ursa in each iteration (a) with and (b) without preprocessing. As Figure 9(a) shows, with preprocessing, almost all iterations finish within half a minute. After closer inspection, we find that most of the time is spent in the ILP solver. While each invocation only takes less than 5 seconds, one iteration consists of up to 20 invocations. We note that while the user inspects causes (which takes 265 seconds on 


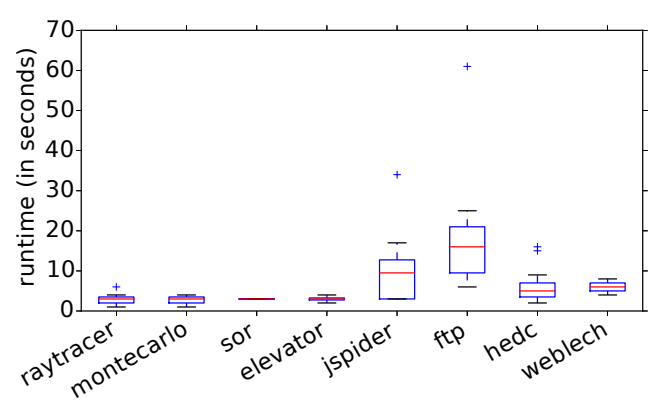

(a) With preprocessing.

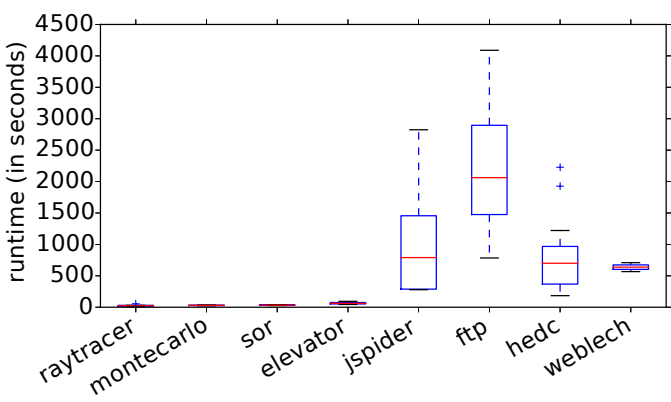

(b) Without preprocessing.

Fig. 9. Time consumed by URSA in each iteration.

average), URSA would have time to speculate what the answers will be, and prepare the next set of questions accordingly. There are two cases in which URSA spent more than half a minute: the first iterations of jspider and ftp. This iteration happens after the static analysis runs and before the user interaction starts. So, from the point of view of the user, it is the same as having a slightly longer static analysis time. Moreover, on the smaller four benchmarks, each iteration of URSA takes only a few seconds. Such fast response times ensure URSA's usability in an interactive setting.

The fact that the Optimum Root Set problem is NP-complete (Section 3.4) precludes us from using approaches with lower complexity than ILP. Nevertheless, we significantly reduce solving time by employing preprocessing techniques that exploit domain knowledge. As a comparison, URSA takes significantly more time per iteration without preprocessing, which is shown in Figure 9(b). While each iteration of URSA across all benchmarks consumes an average time of 826 seconds without preprocessing, it only consumes 8 seconds with preprocessing. The average speedup with preprocessing is as high as $62 \times$ and the maximum speedup goes up to $202 \times$ (from 1,011 seconds to 5 seconds). The effectiveness of preprocessing is also reflected by the reduction in problem sizes: the largest ILP instance reduces from 7 million variables and 17 million constraints to 0.1 million variables and 0.3 million constraints. Therefore, we conclude that the preprocessing techniques are essential to URSA's efficiency.

Effect of different heuristics. The results of running the datarace analysis under each heuristic (Section 5) are shown in Figure 10: (a) generalization and (b) prioritization.

Generalization. We begin with a basic sanity check. By Lemma 4.3, if a heuristic returns a subset of potential causes, then it cannot resolve more false alarms. Thus, aggregated can never resolve more false alarms than static_optimistic or dynamic, and static_optimistic can never resolve more false alarms than static_pessimistic. This is indeed the case, as we can see from the white bars in Figure 10(a).

We make two main observations: (i) dynamic has wide variation in performance, and (ii) aggregated has the best average performance. As before, we measure generalization performance by payoff. The variation in payoff under the dynamic heuristic can be explained by a variation in test coverage: dynamic performs well for montecarlo and elevator (which have good test coverage), and poorly for raytracer and hedc (which have poor test coverage). The good average payoff of aggregated shows that aggressive heuristics guide our approach towards asking good questions.

Prioritization. Figure 10(b) shows the prioritization result for each instantiation on raytracer. For comparison, we also show the result with a baseline Heuristic instantiation which returns all tuples in the shared and parallel relations as potential causes. Instantiation aggregated yields the best result: the first six questions posed under it are same as those under the ideal case, and 




(a) Overall for each benchmark.

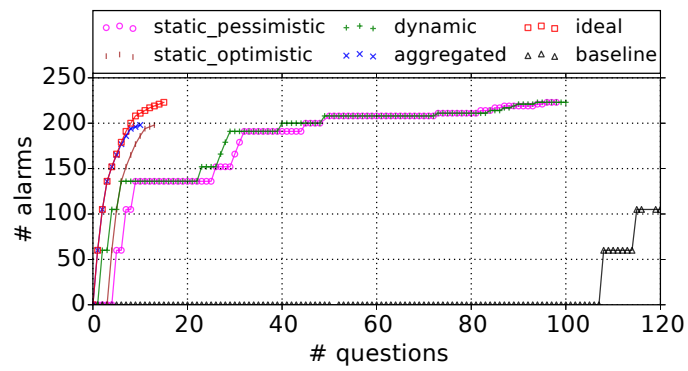

(b) Across iterations on raytracer.

Fig. 10. Number of questions asked and number of false alarms eliminated by URSA with different Heuristics.

answering these questions resolves 177 out of 226 false alarms. The other instantiations perform slightly worse but still prioritize the top three questions in terms of payoff in the first 10 questions. On the other hand, the baseline fails to resolve any alarms for the first 107 iterations, highlighting the effectiveness of using any of our Heuristic instantiations over using none of them.

\section{RELATED WORK}

We survey techniques for reducing user effort in inspecting static analysis alarms. Then, we explain the relation with techniques for combining analyses.

Models for interactive analysis. Different usage models have been proposed to counter the impact of approximations [Dillig et al. 2012; Kremenek et al. 2004; Mangal et al. 2015] or missing specifications [Bastani et al. 2015; Zhu et al. 2013] on the accuracy of static analyses.

In the approaches proposed by Kremenek et al. [2004] and Mangal et al. [2015], user annotations on inspected alarms are generalized to re-rank or re-classify the remaining alarms. These approaches are probabilistic and do not guarantee soundness. Also, users inspect alarm reports rather than intermediate causes.

The technique proposed by Dillig et al. [2012] asks users to resolve intermediate causes that are guaranteed to resolve individual alarms soundly. It aims to minimize user effort needed to resolve a single alarm. In contrast, our approach aims to minimize user effort to resolve all alarms considered together, making it more suitable for static analyses that report large numbers of alarms with shared underlying causes of imprecision.

Instead of approximations, the approaches proposed by Bastani et al. [2015] and Zhu et al. [2013] target specifications for missing program parts that result in mis-classified alarms. In particular, 
they infer candidate specifications that are needed to verify a given assertion, and present them to users for validation. These techniques are applicable to analyses based on standard graph reachability [Zhu et al. 2013] or CFL reachability [Bastani et al. 2015], while we target analyses specified in Datalog.

Just-in-time analysis aims at low-hanging fruit: it first presents alarms that can be found quickly, are unlikely to be false positives, and involve only code close to where the programmer edits. While the programmer responds to these alarms, the analysis searches for more alarms, which are harder to find. A just-in-time analysis exploits user input only in a limited sense: it begins by looking at the code in the programmer's working set. Do et al. [2017] show how to transform an analysis based on the IFDS/IDE framework into a just-in-time analysis. By comparison, our approach targets Datalog (rather than IFDS/IDE), requires a delay in the beginning to run the underlying analysis, but then fully exploits user input and provenance information to minimize the number of alarms presented to the user.

The Ivy model checker takes user guidance to infer inductive invariants [Padon et al. 2016]. Our approach is to start with an over-approximation and ask for user help to prune it down towards the ground truth; the Ivy approach is to guess a separating frontier between reachable and error states and ask for user help in refining this guess both downwards and upwards, until no transition crosses the frontier. Ivy targets small, modeling languages, rather than full-blown programming languages like Java.

Solvers for interactive analysis. Various solving techniques have been developed to incorporate user feedback into analyses or to ask relevant questions to analysis users. Abductive inference, used by Dillig et al. [2012] and Zhu et al. [2013], involves computing minimum satisfying assignments to SMT formulae [Dillig et al. 2012] or minimum-size prime implicants of Boolean formulae [Zhu et al. 2013]. The maximum satisfiability problem used by Mangal et al. [2015] involves solving a system of mixed hard and soft constraints. While the hard constraints encode soundness conditions, the soft constraints encode various objectives such as the costs of different user questions, likelihood of different outcomes, and confidence in different hypotheses. These problems are NP-hard; in contrast, the CFL reachability algorithm used by Bastani et al. [2015] is polynomial time.

Report ranking and clustering. A common approach for reducing a user's effort in inspecting static analysis alarms is to rank them by their likelihood of being true [Blackshear and Lahiri 2013; Jung et al. 2005; Kremenek and Engler 2003]. In the work by Jung et al. [2005], a statistical post-analysis is presented that computes the probability of each alarm being true. Z-ranking [Kremenek and Engler 2003] employs a simple statistical model based on semantic inconsistency detection [Hallem et al. 2002] to rank those alarms most likely to be true errors over those that are least likely. In the work by Blackshear and Lahiri [2013], a post-processing technique also based on semantic inconsistency detection is proposed to de-prioritize reporting alarms in modular verifiers that are caused by overly demonic environments.

Report clustering techniques [Kremenek et al. 2004; Le and Soffa 2010; Lee et al. 2012] group related alarms together to reduce user effort in inspecting redundant alarms. These techniques either compute logical dependencies between alarms [Le and Soffa 2010; Lee et al. 2012], or employ a probabilistic model for finding correlated alarms [Kremenek et al. 2004]. Unlike these techniques, our approach correlates alarms by identifying their common root causes, which are obtained by analyzing the abstract semantics of the analysis at hand.

Our approach is complementary to the above techniques; information such as the truth likelihood of alarms and logical dependencies between alarms can be exploited to further reduce user effort in our approach. 
Spectrum-based fault localization. There is a large body of work on fault localization, especially spectrum-based techniques [Ball et al. 2003; Jones and Harrold 2005; Jones et al. 2002; Liblit et al. 2005; Renieris and Reiss 2003] that seek to pin-point the root causes of failing tests in terms of program behaviors that differ in passing tests, and thereby reduce programmers' debugging effort. Analogously, our approach aims to identify the root causes of alarms, albeit with two crucial differences: our approach is not aware upfront whether each alarm is true or false, unlike in the case of fault localization where each test is known to be failing or passing; secondly, our approach operates on an abstract program semantics used by the given static analysis, whereas fault localization techniques operate on the concrete program semantics.

Interactive program optimization. IOpt [von Dincklage and Diwan 2009, 2011] is an interactive program optimizer. While it targets program performance rather than correctness, it also uses benefit-cost ratios to rank the questions, where the benefit is the expected program speedup and the cost is the estimated user effort in answering the question. However, the underlying techniques to compute such ratios are radically different: while IOpt's approach is based on heuristics, our approach solves a rigorous optimization problem that is generated from the analysis semantics.

Combining multiple analyses. Our method allows a user and an analysis to work together interactively. It is possible to replace the user with another analysis, thus obtaining a method for combining two analyses. We note that the converse is often false: If one starts with a method for combining analyses, then it is usually impossible to replace one of the analyses with the user. The reason is that humans need an interface with low bandwidth. In our case, they get simple yes/no questions. But, if we look at the approach proposed by Zhang et al. [2014], which is closest to our work on a technical level, then we see a tight integration between analyses, in which most state is shared, and it is even difficult to say where the interface between analyses lies. Users are not likely to be effective when presented with the entire internal state of an analysis and asked to perform an update on it. Designing a fruitful method for combining analyses becomes significantly more difficult if one adds the constraint that the interaction must be low bandwidth, and such a constraint is necessary if a user is to be involved.

Low-bandwidth methods for combining analyses may prove useful even in the absence of a user because they do not require analyses to be alike. This is speculation, but we can point to a similar situation that is reality: The Nelson-Oppen method for combining decision procedures is wildly successful because it requires only equalities to be communicated [Nelson and Oppen 1979].

Existing methods for combining analyses do not have the low-bandwidth property. In previous works by Naik et al. [2012]; Oh et al. [2016]; Wei et al. [2016], a fast pre-analysis is used to infer a precise and efficient abstraction for a parametric analysis. This pre-analysis can be either an overapproximating static analysis [Oh et al. 2016] or an under-approximating dynamic analysis [Naik et al. 2012; Wei et al. 2016].

\section{CONCLUSION}

We presented an interactive approach to resolve static analysis alarms. The approach encompasses a new methodology that synergistically combines a sound but imprecise analysis with precise but unsound heuristics. In each iteration, it solves the optimum root set problem which finds a set of questions with the highest expected payoff to pose to the user. We presented an efficient solution to this problem based on integer linear programming for a general class of constraint-based analyses. We demonstrated the effectiveness of our approach in practice at eliminating a majority of false alarms by asking only a few questions, and at prioritizing questions with high payoffs. 


\section{A PROOFS}

Lemma 4.1. A sound analysis $C$ can derive the ground truth; that is, True $=\llbracket C \rrbracket_{\text {False }}$.

Proof. By (2), we have $\llbracket C \rrbracket_{\text {False }} \supseteq$ True. By the augmented semantics (Figure 4(d)), we also know that $\llbracket C \rrbracket_{\text {False }}$ and False are disjoint. The result follows.

Lemma 4.2. In Algorithm 1, suppose that Heuristic returns all tuples T. Also, assume (1), (2), and (3). Then, Algorithm 1 returns the true alarms $\mathrm{A} \cap$ True.

Proof. The key invariant is that

$$
\mathrm{F} \subseteq \text { False } \subseteq \mathrm{Q}
$$

The invariant is established by setting $\mathbf{F}:=\emptyset$ and $Q:=\mathrm{T}$. By (1), we know that $Y \subseteq$ True and $N \subseteq$ False, on line 5. It follows that the invariant is preserved by removing $Y$ from $\mathrm{Q}$, on line 6 . It also follows that $\mathbf{F} \cup N \subseteq$ False and, by (2), that $\llbracket C \rrbracket_{\mathrm{F} \cup N} \supseteq$ True. So, the invariant is also maintained by line 7 . We conclude that (4) is indeed an invariant.

For termination, let us start by showing that $|Q \backslash F|$ is nonincreasing. According to lines 6 and 7 , the values of $\mathbf{Q}$ and $\mathbf{F}$ in the next iteration will be $\mathbf{Q}^{\prime}:=\mathrm{Q} \backslash Y$ and $\mathbf{F}^{\prime}:=(\mathbf{Q} \backslash Y) \backslash \llbracket C \rrbracket_{\mathrm{F} \cup N}$. We now show that $\mathbf{F} \subseteq \mathbf{F}^{\prime}$ and $\mathbf{Q}^{\prime} \subseteq \mathrm{Q}$. Consider an arbitrary $f \in \mathbf{F}$. By (4), $f \in \mathbf{Q}$. Using $Y \subseteq$ True and (4), we conclude that $Y$ and $\mathbf{F}$ are disjoint; using the augmented semantics in Figure 4(d), we conclude that $\llbracket C \rrbracket_{\mathrm{F} \cup N}$ and $\mathbf{F}$ are disjoint. Thus, $f \in \mathbf{F}^{\prime}$, and, since $f$ was arbitrary, we conclude $\mathbf{F} \subseteq \mathbf{F}^{\prime}$. For $\mathrm{Q}^{\prime} \backslash \mathrm{Q}$, it suffices to notice that $Y \subseteq$ True.

Now let us show that $|\mathbf{Q} \backslash \mathbf{F}|$ is not only nonincreasing but in fact decreasing. By (3), we know that $\mathbf{R}$ is non-empty, and thus at least one of $Y$ or $N$ is non-empty. If $Y$ is non-empty, then $\mathbf{Q}^{\prime} \subset \mathrm{Q}$. If $N$ is non-empty, then $\mathbf{F} \subset \mathbf{F}^{\prime}$. We can now conclude that Algorithm 1 terminates.

When the main loop terminates, we have $F=Q$. Together with the invariant (4), we obtain that $\mathbf{F}=$ False. By Lemma 4.1, it follows that Algorithm 1 returns $\mathrm{A} \cap$ True.

\section{B ALTERNATIVE USE CASE: COMBINING TWO STATIC ANALYSES}

While our approach targets interactive alarm resolution, it can be also applied to combine two analyses and balance the overall precision and scalability. We can enable this use case by replacing the human user with a precise yet expensive analysis. Such an iterative combination of the two analyses is beneficial as it may take a significant amount of time to apply the precise analysis to resolve all potential causes in the base analysis. URSA allows the precise analysis to resolve only the causes that are relevant to the alarms and focus on causes with high payoffs. We next demonstrate this use case by using combining two pointer analyses as an example. We first describe the base analysis, its notions of alarms and causes, and our implementation of the procedure Heuristic. Then we describe the oracle analysis. Finally, we empirically evaluate our approach.

Base Analysis. Our base pointer analysis is a flow/context-insensitive, field-sensitive, Andersonstyle analysis with on-the-fly call-graph construction [Lhoták 2002]. It uses object allocation sites as the heap abstraction. It comprises 46 rules, 29 input relations, and 18 output relations.

We treat points-to facts (denoted by relation pointsTo) and call-graph edges (denoted by callEdge) as the alarms as they are directly consumed by client analyses built atop PointerAnalysis. Given the large number of tuples in these two relations, we further limit the alarms to tuples in the application (as opposed to library) code. We treat the same set of tuples as the universe of potential causes since the tuples in these two relations are used to derive each other in a recursive manner.

We are not aware of effective static heuristics for pointer analysis alarms that are client-agnostic. Therefore, we only provide a Heuristic instantiation that leverages a dynamic analysis:

dynamic ()$=\{$ pointsTo $(v, o) \mid$ variable $v$ is accessed in the runs and never points to object $o\} \cup$ \{callEdge $(p, m) \mid$ invocation site $p$ is reached and never invokes method $m$ in the runs \} 
Table 3. Numbers of alarms (denoted by $|\mathbf{A}|$ ) and tuples in the universe of potential causes (denoted by $\left|\mathbf{Q}_{U}\right|$ ) of the pointer analysis, where $\mathrm{k}$ stands for thousands.

\begin{tabular}{r|r|r|r|r}
\hline & \multicolumn{3}{|c|}{$|\mathrm{A}|$} & $\mid$ \\
\cline { 2 - 4 } & false & total & false\% & \\
\hline raytracer & 56 & 950 & $5.9 \%$ & 950 \\
montecarlo & 5 & 867 & $0.6 \%$ & 867 \\
sor & 0 & 159 & 0 & 159 \\
elevator & 3 & 369 & $0.8 \%$ & 369 \\
jspider & 925 & $5.1 \mathrm{k}$ & $18.1 \%$ & $5.1 \mathrm{k}$ \\
hedc & $1.2 \mathrm{k}$ & $3.9 \mathrm{k}$ & $29.8 \%$ & $3.9 \mathrm{k}$ \\
ftp & $5.7 \mathrm{k}$ & $12.5 \mathrm{k}$ & $45.5 \%$ & $12.5 \mathrm{k}$ \\
weblech & 440 & $3.9 \mathrm{k}$ & $11.2 \%$ & $3.9 \mathrm{k}$ \\
\hline
\end{tabular}

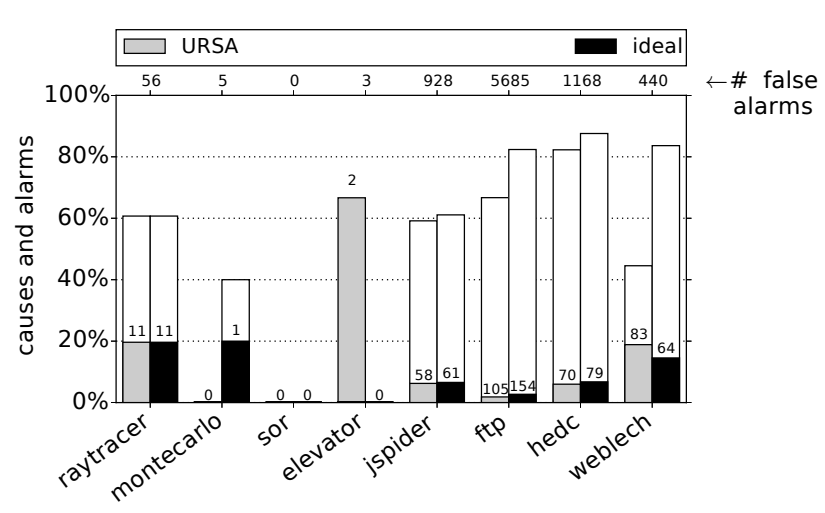

Fig. 11. Number of questions asked over total number of false alarms (denoted by the lower dark bars) and percentage of false alarms resolved (denoted by the upper light bars) by URSA for the pointer analysis.

Oracle Analysis. The oracle analysis is a query-driven $k$-object-sensitive pointer analysis [Zhang et al. 2014]. This analysis improves upon the precision of PointerAnalysis by being simultaneously context- and object-sensitive, but it achieves this higher precision by targeting queries of interest, which in our setting are individual alarms and potential causes.

Empirical Evaluation. We use a setting that is similar to the one described in Section 6.1. In particular, to evaluate the effectiveness of our approach in reducing false alarms, we obtained answers to all the alarms and potential causes offline using the oracle analysis. Table 3 shows the statistics of alarms and tuples in the universal of potential causes. We next describe the generalization results and prioritization results of our approach.

Figure 11 shows the generalization results of URSA with dynamic, the only available Heuristic instantiation for PointerAnalysis. To evaluate the effectiveness of dynamic, we also show the results of the ideal case where the oracle answers are used to implement Heuristic.

URSA is able to eliminate $44.8 \%$ of the false alarms with an average payoff of $8 \times$ per question. Excluding the three small benchmarks with under five false alarms each, the gain rises to $63.2 \%$ and the average payoff increases to $11 \times$. These results show that, most of the false alarms can indeed be eliminated by inspecting only a few common root causes. And by applying the expensive oracle analysis only to these few root causes, URSA can effectively improve the precision of the base analysis without significantly increasing the overall runtime.

In the ideal case, URSA eliminates an additional $15.5 \%$ of false alarms. While the improvement is modest for most benchmarks, an additional $39 \%$ false alarms are eliminated on weblech in the ideal 

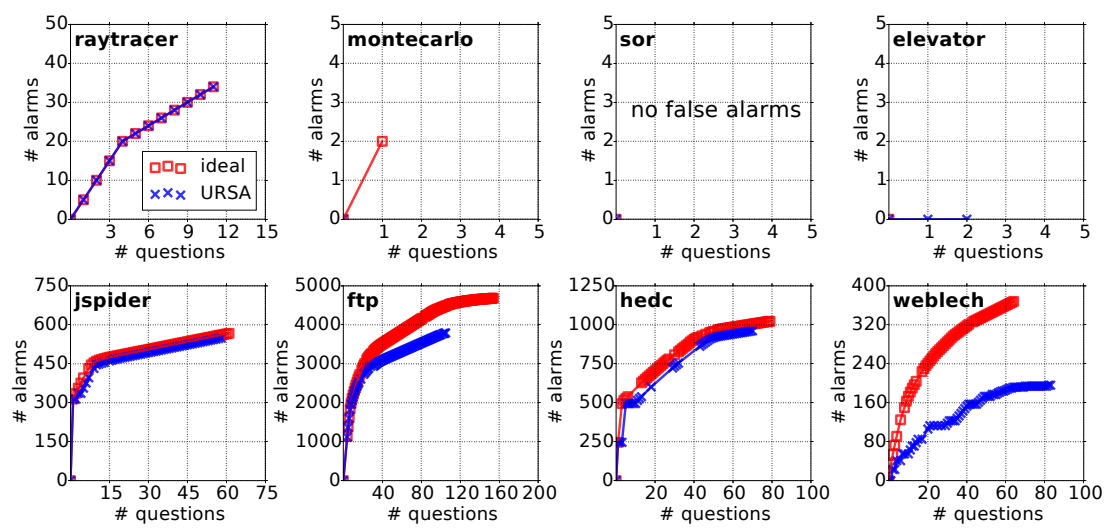

Fig. 12. Number of questions asked and number of false alarms resolved by URSA in each iteration.

case. The reason for this anomaly is that the input set used in dynamic does not yield sufficient code coverage to produce accurate predictions for the desired root causes. We thus conclude that overall, the dynamic instantiation is effective in identifying common root causes of false alarms.

Figure 12 shows the prioritization results of URSA. Every point in the plots represents the number of false alarms eliminated (y-axis) and the number of questions ( $\mathrm{x}$-axis) asked up to the current iteration. As before, we compare the results of URSA to the ideal case.

We observe that a few causes often yield most of the benefits. For instance, four causes can resolve 1,133 out of 5,685 false alarms on $\mathrm{ftp}$, yielding a payoff of $283 \times$. URSA successfully identifies these causes with high payoffs and poses them to the oracle analysis in the earliest few iterations. In fact, for the first three iterations on most benchmarks, URSA asks exactly the same set of questions as the ideal setting. The results of these two settings only differ in later iterations, where the payoff becomes relatively low. We also notice that there can be multiple causes in the set that gives the highest benefit (for instance, the aforementioned ftp results). The reason is that there can be multiple derivations to each alarm. If we naively search the potential causes by fixing the number of questions in advance, we can miss such causes. URSA, on the other hand, successfully finds them by solving the optimal root set problem iteratively.

The fact that URSA is able to prioritize causes with high payoffs allows us to stop the interaction of the two analyses after a few iterations and still get most of the benefits. URSA terminates either when the expected payoff drops to one or when there are no more questions to ask. But in practice, we might choose to stop the interaction even earlier. For instance, we might be satisfied with the current result, or we may find limited payoff in running the oracle analysis, which can take a long time comparing to inspecting the rest alarms manually.

We study these causes with high payoffs more closely. The main causes are the points-to facts and call-graph edges that lead to one or multiple spurious call-graph edges, which in turn lead to many false tuples. Such spurious tuples are produced due to context insensitivity of the analysis.

\section{ACKNOWLEDGMENTS}

We thank Aditya Nori for discussions. We also thank the anonymous reviewers for insightful comments. This work was supported by DARPA under agreement \#FA8750-15-2-0009, NSF awards \#1253867 and \#1526270, MIT Lincoln Laboratory, and a Facebook Fellowship. The U.S. Government is authorized to reproduce and distribute reprints for Governmental purposes notwithstanding any copyright thereon. 


\section{REFERENCES}

2015. UpWork. http://www.upwork.com. (2015). Accessed: 2015-11-19.

Nathaniel Ayewah, David Hovemeyer, J. David Morgenthaler, John Penix, and William Pugh. 2008. Using static analysis to find bugs. IEEE Software (2008).

Thomas Ball, Mayur Naik, and Sriram K. Rajamani. 2003. From symptom to cause: localizing errors in counterexample traces. In POPL.

Osbert Bastani, Saswat Anand, and Alex Aiken. 2015. Specification inference using context-free language reachability. In POPL.

Al Bessey, Ken Block, Benjamin Chelf, Andy Chou, Bryan Fulton, Seth Hallem, Charles-Henri Gros, Asya Kamsky, Scott McPeak, and Dawson R. Engler. 2010. A few billion lines of code later: using static analysis to find bugs in the real world. Commun. ACM (2010).

Sam Blackshear and Shuvendu Lahiri. 2013. Almost-correct specifications: a modular semantic framework for assigning confidence to warnings. In PLDI.

Tom Copeland. 2005. PMD applied. (2005).

Isil Dillig, Thomas Dillig, and Alex Aiken. 2012. Automated error diagnosis using abductive inference. In PLDI.

Lisa Nguyen Quang Do, Karim Ali, Benjamin Livshits, Eric Bodden, Justin Smith, and Emerson Murphy-Hill. 2017. Just-intime static analysis. In ISSTA.

Michael D. Ernst, Jake Cockrell, William G. Griswold, and David Notkin. 2001. Dynamically discovering likely program invariants to support program evolution. IEEE Trans. Software Eng. (2001).

Gurobi Optimization, Inc. 2016. Gurobi optimizer reference manual. http://www.gurobi.com. (2016).

Seth Hallem, Benjamin Chelf, Yichen Xie, and Dawson R. Engler. 2002. A system and language for building system-specific, static analyses. In PLDI.

James A. Jones and Mary Jean Harrold. 2005. Empirical evaluation of the tarantula automatic fault-localization technique. In ASE.

James A. Jones, Mary Jean Harrold, and John T. Stasko. 2002. Visualization of test information to assist fault localization. In ICSE.

Herbert Jordan, Bernhard Scholz, and Pavle Subotic. 2016. Soufflé: on synthesis of program analyzers. In $C A V$.

Yungbum Jung, Jaehwang Kim, Jaeho Shin, and Kwangkeun Yi. 2005. Taming false alarms from a domain-unaware C analyzer by a bayesian statistical post analysis. In $S A S$.

Ted Kremenek, Ken Ashcraft, Junfeng Yang, and Dawson Engler. 2004. Correlation exploitation in error ranking. In FSE.

Ted Kremenek and Dawson Engler. 2003. Z-Ranking: using statistical analysis to counter the impact of static analysis approximations. In SAS.

Wei Le and Mary Lou Soffa. 2010. Path-based Fault Correlations. In FSE.

Woosuk Lee, Wonchan Lee, and Kwangkeun Yi. 2012. Sound non-statistical clustering of static analysis alarms. In VMCAI.

Ondrej Lhoták. 2002. Spark: A flexible points-to analysis framework for Java. (2002).

Ben Liblit, Mayur Naik, Alice X. Zheng, Alexander Aiken, and Michael I. Jordan. 2005. Scalable statistical bug isolation. In PLDI.

Benjamin Livshits, Manu Sridharan, Yannis Smaragdakis, Ondrej Lhoták, José Nelson Amaral, Bor-Yuh Evan Chang, Samuel Z. Guyer, Uday P. Khedker, Anders Møller, and Dimitrios Vardoulakis. 2015. In defense of soundiness: a manifesto. CACM (2015).

Magnus Madsen, Ming-Ho Yee, and Ondrej Lhoták. 2016. From Datalog to Flix: a declarative language for fixed points on lattices. In PLDI.

Ravi Mangal, Xin Zhang, Aditya V. Nori, and Mayur Naik. 2015. A user-guided approach to program analysis. In FSE.

Mayur Naik. 2006. Chord: A Program Analysis Platform for Java. http://jchord.googlecode.com/. (2006).

Mayur Naik, Alex Aiken, and John Whaley. 2006. Effective static race detection for Java. In PLDI.

Mayur Naik, Hongseok Yang, Ghila Castelnuovo, and Mooly Sagiv. 2012. Abstractions from tests. In POPL.

Greg Nelson and Derek C. Oppen. 1979. Simplification by cooperating decision procedures. ACM TOPLAS (1979).

Hakjoo Oh, Wonchan Lee, Kihong Heo, Hongseok Yang, and Kwangkeun Yi. 2016. Selective X-sensitive analysis guided by impact pre-analysis. ACM TOPLAS (2016).

Oded Padon, Kenneth McMillan, Aurojit Panda, Mooly Sagiv, and Sharon Shoham. 2016. Ivy: safety verification by interactive generalization. In PLDI.

Christos H. Papadimitriou. 1981. On the complexity of integer programming. F. ACM (1981).

J. Ross Quinlan. 1993. C4.5: Programs for Machine Learning. Morgan Kaufmann.

Manos Renieris and Steven P. Reiss. 2003. Fault localization with nearest neighbor queries. In ASE.

Henry Gordon Rice. 1953. Classes of recursively enumerable sets and their decision problems. Trans. Amer. Math. Soc. (1953). 
Yannis Smaragdakis and Martin Bravenboer. 2010. Using Datalog for fast and easy program analysis. In Datalog 2.0 Workshop.

Yannis Smaragdakis, George Kastrinis, and George Balatsouras. 2014. Introspective analysis: context-sensitivity, across the board. In PLDI.

Daniel von Dincklage and Amer Diwan. 2009. Optimizing programs with intended semantics. In OOPSLA.

Daniel von Dincklage and Amer Diwan. 2011. Integrating program analyses with programmer productivity tools. Softw. Pract. Exper. (2011).

Shiyi Wei, Omer Tripp, Barbara G. Ryder, and Julian Dolby. 2016. Revamping JavaScript static analysis via localization and remediation of root causes of imprecision. In FSE.

John Whaley, Dzintars Avots, Michael Carbin, and Monica S. Lam. 2005. Using Datalog with binary decision diagrams for program analysis. In APLAS.

Xin Zhang, Ravi Mangal, Radu Grigore, Mayur Naik, and Hongseok Yang. 2014. On abstraction refinement for program analyses in Datalog. In PLDI.

Haiyan Zhu, Thomas Dillig, and Isil Dillig. 2013. Automated inference of library specifications for source-sink property verification. In APLAS 2013. 